Discrete Comput Geom 29:257-277 (2003)

DOI: $10.1007 / \mathrm{s} 00454-002-2837-\mathrm{x}$

\title{
Mixed Volume Computation for Semi-Mixed Systems*
}

\author{
Tangan $\mathrm{Gao}^{1}$ and T. Y. $\mathrm{Li}^{2}$ \\ ${ }^{1}$ Department of Mathematics, California State University, \\ Long Beach, CA 90840, USA \\ tgao@csulb.edu \\ ${ }^{2}$ Department of Mathematics, Michigan State University, \\ East Lansing, MI 48824, USA \\ li@math.msu.edu
}

\begin{abstract}
The Li-Li algorithm produced in [11] for the mixed volume computation of fully mixed polynomial systems is reconstructed in this article for general semi-mixed polynomial systems. Taking the special structure of the semi-mixed supports of the systems into account, the resulting algorithm, illustrated by numerical results, can dramatically speed up the mixed volume computation, especially when the systems are unmixed. Even when applied to fully mixed systems, the new algorithm improves the speed of the $\mathrm{Li}-\mathrm{Li}$ algorithm by a considerable amount.
\end{abstract}

\section{Introduction}

For a system of polynomials $P(\mathbf{x})=\left(p_{1}(\mathbf{x}), \ldots, p_{n}(\mathbf{x})\right)$ with $\mathbf{x}=\left(x_{1}, \ldots, x_{n}\right)$, write

$$
p_{j}(\mathbf{x})=\sum_{\mathbf{a} \in \mathcal{S}_{j}} c_{j, \mathbf{a}} \mathbf{x}^{\mathbf{a}}, \quad j=1, \ldots, n,
$$

where $\mathbf{a}=\left(a_{1}, \ldots, a_{n}\right) \in \mathbb{N}^{n}, c_{j, \mathbf{a}} \in \mathbb{C}^{*}=\mathbb{C} \backslash\{0\}$, and $\mathbf{x}^{\mathbf{a}}=x_{1}^{a_{1}} \cdots x_{n}^{a_{n}}$. Here $\mathcal{S}_{j}$, a finite subset of $\mathbb{N}^{n}$, is called the support of $p_{j}(\mathbf{x})$, and $\mathcal{S}=\left(\mathcal{S}_{1}, \ldots, \mathcal{S}_{n}\right)$ is called the support of $P(\mathbf{x})$.

Let $\mathcal{Q}_{j}=\operatorname{conv}\left(\mathcal{S}_{j}\right)$ for $j=1, \ldots, n$. For positive numbers $\lambda_{1}, \ldots, \lambda_{n}$, the $n$ dimensional volume of the Minkovski sum

$$
\lambda_{1} \mathcal{Q}_{1}+\cdots+\lambda_{n} \mathcal{Q}_{n} \equiv\left\{\lambda_{1} \mathbf{q}_{1}+\cdots+\lambda_{n} \mathbf{q}_{n} \mid \mathbf{q}_{j} \in \mathcal{Q}_{j}, j=1, \ldots, n\right\}
$$

* The research by the second author was supported in part by NSF under Grant DMS-0104009. 
is a homogeneous polynomial of degree $n$ in the variables $\lambda_{1}, \ldots, \lambda_{n}$. The coefficient of $\lambda_{1} \times \cdots \times \lambda_{n}$ in this polynomial is defined to be the mixed volume of $\mathcal{S}=\left(\mathcal{S}_{1}, \ldots, \mathcal{S}_{n}\right)$, denoted by $\mathcal{M}(\mathcal{S})$. On most occasions, we also call $\mathcal{M}(\mathcal{S})$ the mixed volume of $P(\mathbf{x})$.

By Bernshtein's theory [1], the mixed volume $\mathcal{M}(\mathcal{S})$ of $\mathcal{S}=\left(\mathcal{S}_{1}, \ldots, \mathcal{S}_{n}\right)$ of the polynomial system $P(\mathbf{x})=\left(p_{1}(\mathbf{x}), \ldots, p_{n}(\mathbf{x})\right)$ provides an upper bound for the number of isolated zeros in $\left(\mathbb{C}^{*}\right)^{n}$, counting multiplicities. This bound can be reached if the coefficients of $P(\mathbf{x})$ are generic, or the system is in general position. This root count in $\left(\mathbb{C}^{*}\right)^{n}$ has been extended to the root count in $\mathbb{C}^{n}$ [8], [12]-[14]. They are, in general, significantly much sharper than the classical Bézout number and its variants.

Based on this combinatorial root count, the so-called polyhedral homotopies were established recently to approximate all the isolated zeros of $P(\mathbf{x})$ by the homotopy continuation method, yielding a drastic improvement over the classical continuation method by using linear homotopies [7], [9], [10]. When the polyhedral homotopy is employed to find all isolated zeros of $P(\mathbf{x})$, the process of locating all the fine mixed cells in a fine mixed subdivision of the support $\mathcal{S}$ during the mixed volume computation plays a crucially important role: the mixed volume determines the number of solution paths to be traced and the fine mixed cells provide starting points of the solution paths. Calculating the fine mixed cells (and thus the mixed volume) of the support $\mathcal{S}$ consumes a large part of the computation and therefore dictates the efficiency of the method as well as the scope of its applications. Most recently, an efficient algorithm has been produced by $\mathrm{Li}$ and $\mathrm{Li}[11]$ to compute the mixed volume $\mathcal{M}(\mathcal{S})$ by locating all the fine mixed cells in a fine mixed subdivision of the support $\mathcal{S}$. (A similar approach is given in [15].) The algorithm achieves a major advance in speed with much less memory requirement than the existing codes in [4]-[6] and [16].

The polynomial system $P(\mathbf{x})$ is called semi-mixed of type $\left(k_{1}, \ldots, k_{r}\right)$ when the supports $\mathcal{S}_{i}$ 's are not all distinct, they are equal within $r$ blocks of sizes $k_{1}, \ldots, k_{r}$. More precisely, there are $r$ sets $\mathcal{S}^{(1)}, \ldots, \mathcal{S}^{(r)} \subset \mathbb{N}^{n}$ such that

$$
\mathcal{S}^{(i)}=\mathcal{S}_{i 1}=\cdots=\mathcal{S}_{i k_{i}},
$$

where

$$
\mathcal{S}_{i l} \in\left\{\mathcal{S}_{1}, \ldots, \mathcal{S}_{n}\right\}, \quad \text { for } \quad 1 \leq i \leq r, \quad 1 \leq l \leq k_{i},
$$

and $k_{1}+\cdots+k_{r}=n$. The system $P(\mathbf{x})$ is called unmixed if $r=1$ and fully mixed when $r=n$.

The Li-Li algorithm developed in [11] aims mainly at the mixed volume computation of general fully mixed systems, it is not capable of benefiting from the special structure of the supports when the systems are semi-mixed. We show in this paper that if we can take this special structure of the supports into account, the resulting algorithm can dramatically speed up the mixed volume computation for semi-mixed systems, especially when the systems are unmixed. When applied to fully unmixed systems, such as the nine-point problem in mechanism design [18], our algorithm also achieves a considerable speedup over the Dynamic Lifting method in [17], a method well capable of capitalizing the characteristic of all equal supports in mixed volume computation .

When reconstructing the $\mathrm{Li}-\mathrm{Li}$ algorithm, we became aware that a great deal of information generated during the process of the $\mathrm{Li}-\mathrm{Li}$ algorithm was not fully utilized. 
With this important observation, our generalized algorithm improves the speed of the $\mathrm{Li}-$ Li algorithm by a large amount even when applied to fully mixed systems with no special structure in the supports as illustrated by the numerical results exhibited in Section 5. For instance, the CPU times of the $\mathrm{Li}-\mathrm{Li}$ algorithm on the widely considered notoriously difficult cyclic- $n$ root problems are substantially reduced by our algorithm: we only need 6 hours and 32 minutes for the cyclic-13 problem, in contrast to 17 hours and 4 minutes by the $\mathrm{Li}-\mathrm{Li}$ algorithm on the same machine.

Without any modification, the method presented in this paper also works for the Laurent polynomial systems which admit negative integer exponents. We thus assume from here on that $\mathcal{S}^{(i)} \subset \mathbb{Z}^{n}$ for all $i=1, \ldots, r$.

\section{Preliminaries}

By a cell of $\mathcal{S}=\left(\mathcal{S}^{(1)}, \ldots, \mathcal{S}^{(r)}\right)$, where $\mathcal{S}^{(i)}$ is a finite subset of $\mathbb{Z}^{n}$ for each $i=1, \ldots, n$, we mean an $r$-tuple $C=\left(C_{1}, \ldots, C_{r}\right)$ of subsets $C_{i} \subseteq \mathcal{S}^{(i)}$ for $i=1, \ldots, r$. With $\operatorname{dim}\left(C_{i}\right):=\operatorname{dim}\left(\operatorname{conv}\left(C_{i}\right)\right)$, define

$$
\begin{aligned}
\operatorname{type}(C) & :=\left(\operatorname{dim}\left(C_{1}\right), \ldots, \operatorname{dim}\left(C_{r}\right)\right), \\
\operatorname{conv}(C) & :=\operatorname{conv}\left(C_{1}\right)+\cdots+\operatorname{conv}\left(C_{r}\right) .
\end{aligned}
$$

A face of $C=\left(C_{1}, \ldots, C_{r}\right)$ is a subcell $F=\left(F_{1}, \ldots, F_{r}\right)$ of $C$ where $F_{i} \subseteq C_{i}$ and some linear functional $\alpha \in\left(\mathbb{R}^{n}\right)^{\vee}$ attains its minimum over $C_{i}$ at $F_{i}$ for $i=1, \ldots, r$. Such an $\alpha$ is called an inner normal of $F$. (Recall that for a convex polytope $T$ in $\mathbb{R}^{n}$ the linear functional $\alpha \in\left(\mathbb{R}^{n}\right)^{\vee}$ which attains its minimum on a face of $T$ is called an inner normal of the face.) A fine mixed subdivision of $\mathcal{S}=\left(\mathcal{S}^{(1)}, \ldots, \mathcal{S}^{(r)}\right)$ is a set of cells $\left\{C^{(1)}, \ldots, C^{(m)}\right\}$, where $C^{(j)}=\left(C_{1}^{(j)}, \ldots, C_{r}^{(j)}\right), j=1, \ldots, m$, satisfy

(a) $\operatorname{dim}\left(\operatorname{conv}\left(C^{(j)}\right)\right)=n$ for all $j=1, \ldots, m$;

(b) $\operatorname{conv}\left(C^{(j)}\right) \cap \operatorname{conv}\left(C^{(k)}\right)$ is a proper common face of $\operatorname{conv}\left(C^{(j)}\right)$ and $\operatorname{conv}\left(C^{(k)}\right)$, when it is nonempty for $j \neq k$,

(c) $\bigcup_{j=1}^{m} \operatorname{conv}\left(C^{(j)}\right)=\operatorname{conv}(\mathcal{S})$; and

(d) for each $j=1, \ldots, m, \operatorname{conv}\left(C_{i}^{(j)}\right)$ is a simplex of dimension $\# C_{i}^{(j)}-1$ where $\# C_{i}^{(j)}$ stands for the number of points in $C_{i}^{(j)}$, and

$$
\sum_{i=1}^{r} \operatorname{dim}\left(\operatorname{conv}\left(C_{i}^{(j)}\right)\right)=n
$$

For a semi-mixed polynomial system $P(\mathbf{x})=\left(p_{1}(\mathbf{x}), \ldots, p_{n}(\mathbf{x})\right)$ of type $\left(k_{1}, \ldots, k_{r}\right)$ with support $\mathcal{S}=\left(\mathcal{S}^{(1)}, \ldots, \mathcal{S}^{(r)}\right)$, it was shown in [7] that the mixed volume of $\mathcal{S}$ equals the sum of $n$-dimensional volumes of all the cells of type $\left(k_{1}, \ldots, k_{r}\right)$ in a fine mixed subdivision of $\mathcal{S}=\left(\mathcal{S}^{(1)}, \ldots, \mathcal{S}^{(r)}\right)$. A fine mixed subdivision of $\mathcal{S}=\left(\mathcal{S}^{(1)}, \ldots, \mathcal{S}^{(r)}\right)$ can be found by applying a random lifting $\omega=\left(\omega_{1}, \ldots, \omega_{r}\right)$ to $\mathcal{S}$ where image values of $\omega_{i}: \mathcal{S}_{i} \rightarrow \mathbb{R}$ are chosen generically for each $i=1, \ldots, r$ [7]. The lifting $\omega_{i}$ lifts $\mathcal{S}^{(i)}$ to its graph $\hat{\mathcal{S}}^{(i)}=\left\{\left(\mathbf{a}, \omega_{i}(\mathbf{a})\right) \mid \mathbf{a} \in \mathcal{S}^{(i)}\right\} \subset \mathbb{R}^{n+1}$. This notion is extended in the obvious way: $\hat{\mathcal{S}}=\left(\hat{\mathcal{S}}^{(1)}, \ldots, \hat{\mathcal{S}}^{(r)}\right), \hat{\mathcal{Q}}_{i}=\operatorname{conv}\left(\hat{\mathcal{S}}^{(i)}\right), \hat{\mathcal{Q}}=\operatorname{conv}(\hat{\mathcal{S}})=\hat{\mathcal{Q}}_{1}+\cdots+\hat{\mathcal{Q}}_{r}$, etc. Recall 
that a facet of $\hat{\mathcal{Q}}$ is a face of $\hat{\mathcal{Q}}$ of co-dimension 1 and the lower hull of $\hat{\mathcal{Q}}$ consists of all the faces of $\hat{\mathcal{Q}}$ whose inner normals admit a positive last coordinate, such faces are called lower faces of $\hat{\mathcal{Q}}$. We call cell $\hat{C}=\left(\hat{C}_{1}, \ldots, \hat{C}_{r}\right)$ of $\hat{\mathcal{S}}=\left(\hat{\mathcal{S}}^{(1)}, \ldots, \hat{\mathcal{S}}^{(r)}\right)$ a lower face of $\hat{\mathcal{S}}$ when $\operatorname{conv}(\hat{C})$ is in the lower hull of $\hat{\mathcal{Q}}$, and, similarly, $\hat{C}$ is a lower facet of $\hat{\mathcal{S}}$ if $\operatorname{conv}(\hat{C})$ is a lower facet of $\hat{\mathcal{Q}}$. Let $S_{\omega}$ be the set of cells of $\mathcal{S}$ where for each $C \in S_{\omega}$, $\hat{C}$ is a lower facet of $\hat{\mathcal{S}}$. It was shown in [7] that $S_{\omega}$ forms a fine mixed subdivision of $\mathcal{S}$. The cells of type $\left(k_{1}, \ldots, k_{r}\right)$ in $S_{\omega}$ can be found by identifying their corresponding lower facets of $\hat{\mathcal{S}}$ of the same type. Namely, if $\hat{C}=\left(\hat{C}_{1}, \ldots, \hat{C}_{r}\right)$ is a lower facet of $\hat{\mathcal{S}}=\left(\hat{\mathcal{S}}^{(1)}, \ldots, \hat{\mathcal{S}}^{(r)}\right)$ of type $\left(k_{1}, \ldots, k_{r}\right)$, then $C=\left(C_{1}, \ldots, C_{r}\right)$ yields a cell of type $\left(k_{1}, \ldots, k_{r}\right)$ in $S_{\omega}$.

To find all the lower facets of $\hat{\mathcal{S}}=\left(\hat{\mathcal{S}}^{(1)}, \ldots, \hat{\mathcal{S}}^{(r)}\right)$ of type $\left(k_{1}, \ldots, k_{r}\right)$ we must repeatedly deal with LP (linear programming) problems of the following type:

$$
\begin{gathered}
\text { Minimize }\langle\mathbf{f}, \mathbf{z}\rangle \\
\left\langle\mathbf{c}_{i}, \mathbf{z}\right\rangle \leq b_{i}, \quad i=1, \ldots, m,
\end{gathered}
$$

where $\left\{\mathbf{f}, \mathbf{c}_{i}\right\} \subset \mathbb{R}^{n}, \mathbf{b}=\left(b_{1}, \ldots, b_{m}\right)^{T} \in \mathbb{R}^{m}, \mathbf{z}=\left(z_{1}, \ldots, z_{n}\right)$, and $m>n$.

To solve these problems, it is better to employ the classical simplex algorithm instead of using the faster interior point method because our main algorithm for mixed volume computation takes great advantage of the rich information generated by the pivoting process in the simplex method. The algorithm for solving the LP problem in (1) is briefly outlined below. The details can be found in many standard LP textbooks, e.g., [2].

The feasible region of (1), denoted by $R$, defines a polyhedral set. By a nondegenerate vertex of $R$ we mean a feasible point of $R$ with exactly $n$ active constraints. From a feasible point of the problem, or a point in the feasible region of (1), one may always attain a nondegenerate vertex of $R$. Let $\mathbf{z}^{0}$ be such a point and let $J=\left\{j_{1}, \ldots, j_{n}\right\}$ be the set of indices of currently active constraints at $\mathbf{z}^{0}$, that is,

$$
\begin{aligned}
& \left\langle\mathbf{c}_{i}, \mathbf{z}^{0}\right\rangle=b_{i}, \quad \text { if } \quad i \in J, \\
& \left\langle\mathbf{c}_{i}, \mathbf{z}^{0}\right\rangle<b_{i}, \quad \text { if } \quad i \notin J .
\end{aligned}
$$

Let $D^{T}=\left[\mathbf{c}_{j_{1}}, \ldots, \mathbf{c}_{j_{n}}\right]$. Since $\mathbf{z}^{0}$ is a nondegenerate vertex point, $D$ must be nonsingular, so let $D^{-1}=\left[\mathbf{u}_{1}, \ldots, \mathbf{u}_{n}\right]$.

It can be shown that the $n$ edges of the feasible region $R$ emanating from $\mathbf{z}^{0}$ can be represented in the form

$$
\mathbf{z}^{0}-\sigma \mathbf{u}_{k}, \quad \sigma>0, \quad k=1, \ldots, n .
$$

Along all those edges, the objective function $\left\langle\mathbf{f}, \mathbf{z}^{0}-\sigma \mathbf{u}_{k}\right\rangle$ decreases as a function of $\sigma>0$ when $\left\langle\mathbf{f}, \mathbf{u}_{k}\right\rangle>0$. For such a direction $\mathbf{u}_{k}$, the largest possible $\sigma>0$ for $\mathbf{z}^{0}-\sigma \mathbf{u}_{k}$ to stay feasible is

$$
\sigma_{0}=\min \left\{\frac{\left\langle\mathbf{c}_{i}, \mathbf{z}^{0}\right\rangle-b_{i}}{\left\langle\mathbf{c}_{i}, \mathbf{u}_{k}\right\rangle} \mid i \notin J \text { with }\left\langle\mathbf{c}_{i}, \mathbf{u}_{k}\right\rangle<0\right\}
$$

and the point $\mathbf{z}^{1}=\mathbf{z}^{0}-\sigma_{0} \mathbf{u}_{k}$ yields an adjacent vertex of the feasible region $R$ with a reduced objective function value. 
It is easy to see that $\mathbf{z}^{0}$ is an optimal solution of (1) if $\left\langle\mathbf{f}, \mathbf{u}_{i}\right\rangle \leq 0$ for all $i=1, \ldots, n$. Hence we may solve the LP problem in (1) directly by moving from one vertex to an adjacent one in the direction where the objective function decreases until a vertex with $\left\langle\mathbf{f}, \mathbf{u}_{i}\right\rangle \leq 0$ for all $i=1, \ldots, n$ is reached. On the other hand, if for all $k$ where $\left\langle\mathbf{f}, \mathbf{u}_{k}\right\rangle>0,\left\langle\mathbf{c}_{i}, \mathbf{u}_{k}\right\rangle$ are nonnegative for all $i$, then the problem is unbounded and the solution does not exist.

Most frequently, the LP problems arising in our algorithm belong to the following type:

$$
\begin{aligned}
\operatorname{Minimize} & \langle\mathbf{f}, \mathbf{z}\rangle \\
\left\langle\mathbf{a}_{i}, \mathbf{z}\right\rangle & =b_{i}, \quad \\
\left\langle\mathbf{c}_{j}, \mathbf{z}\right\rangle \leq b_{j}, & i \in I_{1}=\{1, \ldots, q\},
\end{aligned}
$$

where $\left\{\mathbf{f}, \mathbf{a}_{i}, \mathbf{c}_{j}\right\} \subset \mathbb{R}^{n},\left(b_{1}, \ldots, b_{m}\right)^{T} \in \mathbb{R}^{m}$, and $q<n<m$. In such situations, we may turn this problem into the LP problem in (1) by eliminating the equality constraints via reducing an equal number of variables in $\mathbf{z}=\left(z_{1}, \ldots, z_{n}\right)$. For instance,

$$
\begin{aligned}
\left\langle\mathbf{a}_{1}, \mathbf{z}\right\rangle & =b_{1}, \\
& \vdots \\
\left\langle\mathbf{a}_{q}, \mathbf{z}\right\rangle & =b_{q}
\end{aligned}
$$

implies, without loss of generality,

$$
\begin{array}{ccc}
z_{1} & & +a_{1, q+1}^{\prime} z_{q+1}+\cdots+a_{1, n}^{\prime} z_{n}=b_{1}, \\
& \ddots & \vdots \\
& z_{q}+a_{q, q+1}^{\prime} z_{q+1}+\cdots+a_{q, n}^{\prime} z_{n}=b_{q} .
\end{array}
$$

Solving $\left(z_{1}, \ldots, z_{q}\right)$ in terms of $\left(z_{q+1}, \ldots, z_{n}\right)$ in the above equations and substituting them into the inequality constraints in (2), the LP problem in (2) then becomes

$$
\begin{gathered}
\text { Minimize } \quad\left\langle\mathbf{f}^{\prime}, \mathbf{y}\right\rangle \\
\left\langle\mathbf{c}_{j}^{\prime}, \mathbf{y}\right\rangle \leq b_{j}^{\prime}, \quad j \in I_{2},
\end{gathered}
$$

in the variables $\mathbf{y}=\left(z_{q+1}, \ldots, z_{n}\right)$, where $\left\{\mathbf{f}^{\prime}, \mathbf{c}_{j}^{\prime}\right\} \subset \mathbb{R}^{n-q}$, and $\mathbf{b}=\left(b_{q+1}^{\prime}, \ldots, b_{m}^{\prime}\right)^{T} \in$ $\mathbb{R}^{m-q}$.

\section{The Relation Table}

For each $\mathcal{S}^{(i)} \subset \mathbb{Z}^{n}, i=1, \ldots, r$, it is well known that the interior points of $\mathcal{S}^{(i)}$, or nonvertex points of $\mathcal{Q}_{i}=\operatorname{conv}\left(\mathcal{S}^{(i)}\right)$, play no role in the mixed volume of $\mathcal{S}=$ $\left(\mathcal{S}^{(1)}, \ldots, \mathcal{S}^{(r)}\right)$. Those interior points can be easily identified and removed before the mixed volume computation [11]. We therefore assume that $\mathcal{S}^{(i)}$ admits only extreme points, or noninterior points, for each $i=1, \ldots, r$.

For generic $\omega_{i}: \mathcal{S}^{(i)} \rightarrow \mathbb{R}$ and $\hat{\mathcal{S}}^{(i)}=\left\{\hat{\mathbf{a}}^{(i)}=\left(\mathbf{a}^{(i)}, \omega_{i}\left(\mathbf{a}^{(i)}\right)\right) \mid \mathbf{a}^{(i)} \in \mathcal{S}^{(i)}\right\}$ for each $i=1, \ldots, r$, an important primary step of our algorithm for computing the mixed 
volume of $\mathcal{S}$ is to complete the relation table consisting of pairwise relation subtables $\mathrm{T}(i, j)$ between $\hat{\mathcal{S}}^{(i)}$ and $\hat{\mathcal{S}}^{(j)}$ for all $1 \leq i \leq j \leq r$ as shown in Table 1 . The table $\mathrm{T}(i, j)$ displays the relationships between elements of $\hat{\mathcal{S}}^{(i)}$ and $\hat{\mathcal{S}}^{(j)}$ in the following sense:

Given elements $\hat{\mathbf{a}}^{(i)} \in \hat{\mathcal{S}}^{(i)}$ and $\hat{\mathbf{a}}^{(j)} \in \hat{\mathcal{S}}^{(j)}$ where $\hat{\mathbf{a}}^{(i)} \neq \hat{\mathbf{a}}^{(j)}$ when $i=j$, does there exist an $\hat{\alpha}=(\alpha, 1) \in\left(\mathbb{R}^{n+1}\right)^{\vee}$ such that

$$
\begin{aligned}
& \left\langle\hat{\mathbf{a}}^{(i)}, \hat{\alpha}\right\rangle \leq\left\langle\hat{\mathbf{a}}^{(i)^{\prime}}, \hat{\alpha}\right\rangle, \quad \forall \hat{\mathbf{a}}^{(i)^{\prime}} \in \hat{\mathcal{S}}^{(i)}, \\
& \left\langle\hat{\mathbf{a}}^{(j)}, \hat{\alpha}\right\rangle \leq\left\langle\hat{\mathbf{a}}^{(j)^{\prime}}, \hat{\alpha}\right\rangle, \quad \forall \hat{\mathbf{a}}^{(j)^{\prime}} \in \hat{\mathcal{S}}^{(j)} ?
\end{aligned}
$$

Denote the entry on table $\mathrm{T}(i, j)$ located at the intersection of the row containing $\hat{\mathbf{a}}_{l}^{(i)}$ and the column containing $\hat{\mathbf{a}}_{m}^{(j)}$ by $\left[\hat{\mathbf{a}}_{l}^{(i)}, \hat{\mathbf{a}}_{m}^{(j)}\right]$. We set $\left[\hat{\mathbf{a}}_{l}^{(i)}, \hat{\mathbf{a}}_{m}^{(j)}\right]=1$ when the answer to problem (4) for $\hat{\mathbf{a}}_{l}^{(i)}$ and $\hat{\mathbf{a}}_{m}^{(j)}$ is positive and $\left[\hat{\mathbf{a}}_{l}^{(i)}, \hat{\mathbf{a}}_{m}^{(j)}\right]=0$ otherwise. When $i=j$, it is obvious that $\left[\hat{\mathbf{a}}_{l}^{(i)}, \hat{\mathbf{a}}_{m}^{(i)}\right]=\left[\hat{\mathbf{a}}_{m}^{(i)}, \hat{\mathbf{a}}_{l}^{(i)}\right]$ if $l \neq m$, we therefore always assume $m>l$ for the notation $\left[\hat{\mathbf{a}}_{l}^{(i)}, \hat{\mathbf{a}}_{m}^{(i)}\right]$.

To fill out the relation table, Table 1, we first fix $\hat{\mathbf{a}}_{1}^{(1)}$ on the first row

$$
\hat{\mathbf{a}}{ }_{1}^{(1)}: \overbrace{\left[\hat{\mathbf{a}}_{1}^{(1)}, \hat{\mathbf{a}}_{2}^{(1)}\right], \ldots,\left[\hat{\mathbf{a}}_{1}^{(1)}, \hat{\mathbf{a}}_{s_{1}}^{(1)}\right]}^{\mathrm{T}(1,1)}, \ldots, \overbrace{\left[\hat{\mathbf{a}}_{1}^{(1)}, \hat{\mathbf{a}}_{1}^{(r)}\right], \ldots,\left[\hat{\mathbf{a}}_{1}^{(1)}, \hat{\mathbf{a}}_{s_{r}}^{(r)}\right]}^{\mathrm{T}(1, r)} .
$$

To determine $\left[\hat{\mathbf{a}}_{1}^{(1)}, \hat{\mathbf{a}}_{2}^{(1)}\right]$, we use the One-Point test (similar in spirit to the One-Point test introduced in [11]) consisting of the LP problem:

$$
\begin{aligned}
& \operatorname{Minimize}\left\langle\hat{\mathbf{a}}_{2}^{(1)}, \hat{\alpha}\right\rangle-\alpha_{0} \\
& \quad \alpha_{0}=\left\langle\hat{\mathbf{a}}_{1}^{(1)}, \hat{\alpha}\right\rangle, \\
& \alpha_{0} \leq\left\langle\hat{\mathbf{a}}_{k}^{(1)}, \hat{\alpha}\right\rangle, \quad \forall k \in\left\{2, \ldots, s_{1}\right\},
\end{aligned}
$$

in the variables $\hat{\alpha}=(\alpha, 1) \in\left(\mathbb{R}^{n+1}\right)^{\vee}$ and $\alpha_{0} \in \mathbb{R}$ to test if the point, the One-Point, $\hat{\mathbf{a}}_{2}^{(1)}$ combined with $\hat{\mathbf{a}}_{1}^{(1)}$ as a pair satisfies condition (4). More explicitly, we may rewrite this problem in the form of (1) in Section 2:

$$
\begin{aligned}
& \operatorname{Minimize}\left\langle\mathbf{a}_{2}^{(1)}-\mathbf{a}_{1}^{(1)}, \alpha\right\rangle+\omega_{1}\left(\mathbf{a}_{2}^{(1)}\right)-\omega_{1}\left(\mathbf{a}_{1}^{(1)}\right) \\
& \quad\left\langle\mathbf{a}_{1}^{(1)}-\mathbf{a}_{k}^{(1)}, \alpha\right\rangle \leq \omega_{1}\left(\mathbf{a}_{k}^{(1)}\right)-\omega_{1}\left(\mathbf{a}_{1}^{(1)}\right), \quad \forall k \in\left\{2, \ldots, s_{1}\right\} .
\end{aligned}
$$

Since $\mathbf{a}_{1}^{(1)}$ is a vertex point of $\mathcal{Q}_{1}=\operatorname{conv}\left(\mathcal{S}^{(1)}\right), \hat{\mathbf{a}}_{1}^{(1)}$ must be in the lower hull of $\hat{\mathcal{Q}}_{1}=\operatorname{conv}\left(\hat{\mathcal{S}}^{(1)}\right)$, and any hyperplane in the form $\hat{\alpha}=(\alpha, 1) \in\left(\mathbb{R}^{n+1}\right)^{\vee}$ that supports $\hat{\mathbf{a}}_{1}^{(1)}$ in $\hat{\mathcal{Q}}_{1}$ decides a feasible point of the constraints in (6). Such a feasible point can be obtained by solving a standard Phase I problem in linear programming for the constraints in (7):

Minimize $\varepsilon$

$$
\begin{aligned}
\left\langle\mathbf{a}_{1}^{(1)}-\mathbf{a}_{k}^{(1)}, \alpha\right\rangle-\varepsilon & \leq \omega_{1}\left(\mathbf{a}_{k}^{(1)}\right)-\omega_{1}\left(\mathbf{a}_{1}^{(1)}\right), \quad \forall k \in\left\{2, \ldots, s_{1}\right\}, \\
-\varepsilon & \leq 0
\end{aligned}
$$


Table T $(i, i)$

\begin{tabular}{|c|c|c|c|c|c|}
\hline \multirow{6}{*}{$\hat{\mathbf{a}}_{1}^{(i)}$} & $\hat{\mathbf{a}}_{2}^{(i)}$ & $\hat{\mathbf{a}}_{3}^{(i)}$ & $\cdots$ & $\begin{array}{l}\hat{\mathbf{a}}_{s_{i}-1}^{(i)} \\
\end{array}$ & $\hat{\mathbf{a}}_{s_{i}}^{(i)}$ \\
\hline & {$\left[\hat{\mathbf{a}}_{1}^{(i)}, \hat{\mathbf{a}}_{2}^{(i)}\right]$} & {$\left[\hat{\mathbf{a}}_{1}^{(i)}, \hat{\mathbf{a}}_{3}^{(i)}\right]$} & $\ldots$ & {$\left[\hat{\mathbf{a}}_{1}^{(i)}, \hat{\mathbf{a}}_{s_{i}-1}^{(i)}\right]$} & {$\left[\hat{\mathbf{a}}_{1}^{(i)}, \hat{\mathbf{a}}_{s_{i}}^{(i)}\right]$} \\
\hline & $\hat{\mathbf{a}}_{2}^{(i)}$ & {$\left[\hat{\mathbf{a}}_{2}^{(i)}, \hat{\mathbf{a}}_{3}^{(i)}\right]$} & $\cdots$ & {$\left[\hat{\mathbf{a}}_{2}^{(i)}, \hat{\mathbf{a}}_{s_{i}-1}^{(i)}\right]$} & {$\left[\hat{\mathbf{a}}_{2}^{(i)}, \hat{\mathbf{a}}_{s_{i}}^{(i)}\right]$} \\
\hline & & & $\ddots$ & $\vdots$ & $\vdots$ \\
\hline & & & & $\begin{array}{l}\hat{\mathbf{a}}_{s_{i}-1}^{(i)} \\
\end{array}$ & {$\left[\hat{\mathbf{a}}_{s_{i}-1}^{(i)}, \hat{\mathbf{a}}_{s_{i}}^{(i)}\right]$} \\
\hline & & & & & $\hat{\mathbf{a}}_{s_{i}}^{(i)}$ \\
\hline
\end{tabular}

Table T $(i, j)$

\begin{tabular}{|c|c|c|c|c|c|}
\hline & $\hat{\mathbf{a}}_{1}^{(j)}$ & $\hat{\mathbf{a}}_{2}^{(j)}$ & $\hat{\mathbf{a}}_{3}^{(j)}$ & $\ldots$ & $\hat{\mathbf{a}}_{s_{j}}^{(j)}$ \\
\hline$\hat{\mathbf{a}}_{1}^{(i)}$ & {$\left[\hat{\mathbf{a}}_{1}^{(i)}, \hat{\mathbf{a}}_{1}^{(j)}\right]$} & {$\left[\hat{\mathbf{a}}_{1}^{(i)}, \hat{\mathbf{a}}_{2}^{(j)}\right]$} & {$\left[\hat{\mathbf{a}}_{1}^{(i)}, \hat{\mathbf{a}}_{3}^{(j)}\right]$} & $\ldots$ & {$\left[\hat{\mathbf{a}}_{1}^{(i)}, \hat{\mathbf{a}}_{s_{j}}^{(j)}\right]$} \\
\hline$\hat{\mathbf{a}}_{2}^{(i)}$ & {$\left[\hat{\mathbf{a}}_{2}^{(i)}, \hat{\mathbf{a}}_{1}^{(j)}\right]$} & {$\left[\hat{\mathbf{a}}_{2}^{(i)}, \hat{\mathbf{a}}_{2}^{(j)}\right]$} & {$\left[\hat{\mathbf{a}}_{2}^{(i)}, \hat{\mathbf{a}}_{3}^{(j)}\right]$} & $\cdots$ & {$\left[\hat{\mathbf{a}}_{2}^{(i)}, \hat{\mathbf{a}}_{s_{j}}^{(j)}\right]$} \\
\hline${ }^{\circ}$ & $\vdots$ & : & $\vdots$ & $\ldots$ & $\vdots$ \\
\hline$\hat{\mathbf{a}}_{s_{i}}^{(i)}$ & {$\left[\hat{\mathbf{a}}_{s_{i}}^{(i)}, \hat{\mathbf{a}}_{1}^{(j)}\right]$} & {$\left[\hat{\mathbf{a}}_{s_{i}}^{(i)}, \hat{\mathbf{a}}_{2}^{(j)}\right]$} & {$\left[\hat{\mathbf{a}}_{s_{i}}^{(i)}, \hat{\mathbf{a}}_{3}^{(j)}\right]$} & $\ldots$ & {$\left[\hat{\mathbf{a}}_{s_{i}}^{(i)}, \hat{\mathbf{a}}_{s_{j}}^{(j)}\right]$} \\
\hline
\end{tabular}

Table 1. The relation table.

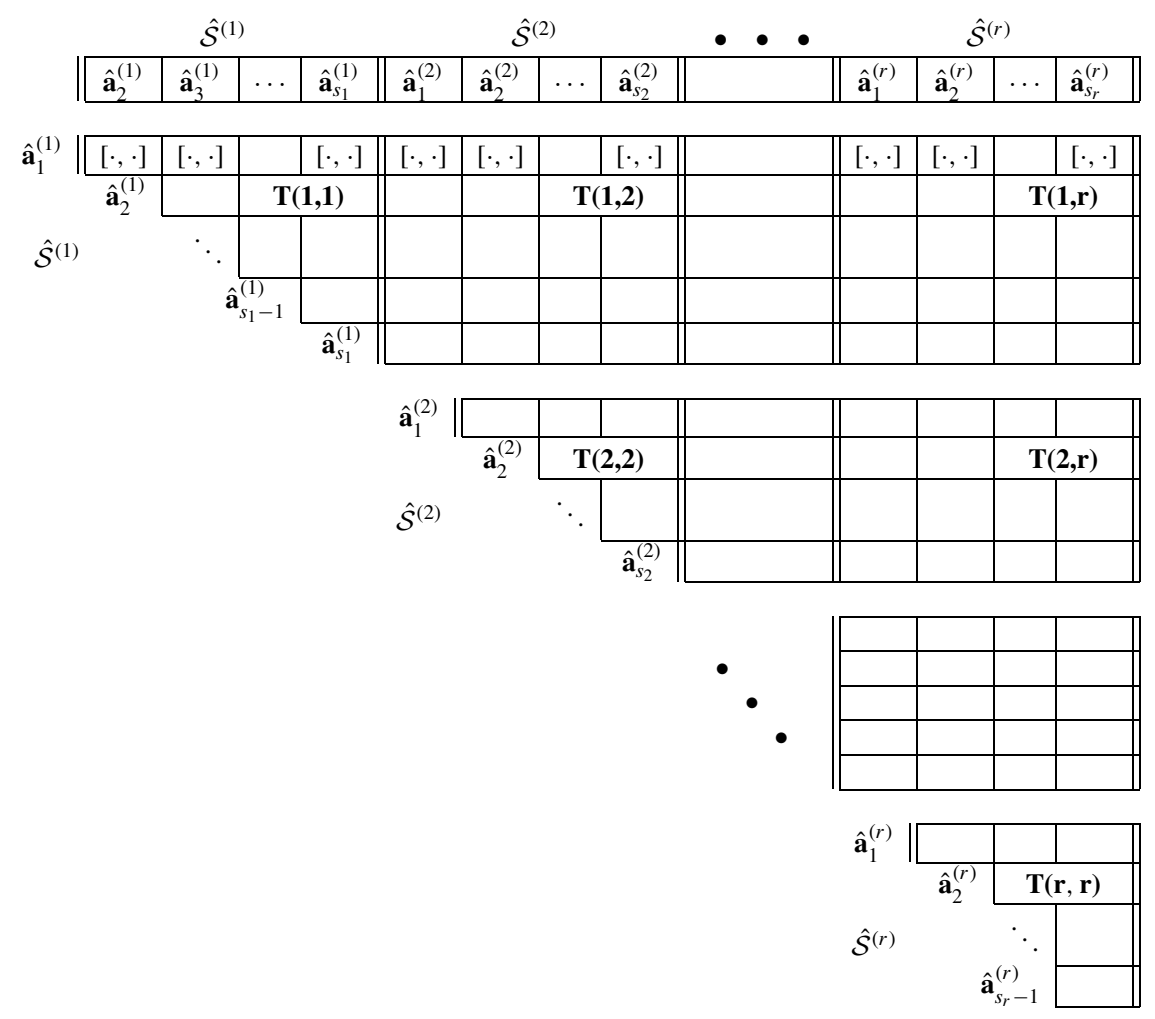


in the variables $\alpha \in\left(\mathbb{R}^{n}\right)^{\vee}$ and $\varepsilon \geq 0$ with a feasible point $\alpha=0$ along with a large enough $\varepsilon>0$.

If the optimal value of the LP problem (6) is zero, then at the optimal solution $\left(\hat{\alpha}, \alpha_{0}\right)$,

$$
\left\langle\hat{\mathbf{a}}_{1}^{(1)}, \hat{\alpha}\right\rangle=\left\langle\hat{\mathbf{a}}_{2}^{(1)}, \hat{\alpha}\right\rangle \leq\left\langle\hat{\mathbf{a}}_{k}^{(1)}, \hat{\alpha}\right\rangle, \quad \forall k \in\left\{3, \ldots, s_{1}\right\} .
$$

This makes $\left[\hat{\mathbf{a}}_{1}^{(1)}, \hat{\mathbf{a}}_{2}^{(1)}\right]=1$. Otherwise, $\left[\hat{\mathbf{a}}_{1}^{(1)}, \hat{\mathbf{a}}_{2}^{(1)}\right]$ must be zero, for if there exists $\hat{\alpha}^{\prime}=(\alpha, 1) \in\left(\mathbb{R}^{n+1}\right)^{\vee}$ for which the inequalities in (8) hold, then this $\hat{\alpha}^{\prime}$ together with $\hat{\alpha}_{0}^{\prime}=\left\langle\hat{\mathbf{a}}_{1}^{(1)}, \hat{\alpha}^{\prime}\right\rangle$ yields a feasible point of (6) at which the objective function value is zero.

When the simplex method is used to solve the LP problem, the pivoting process in the algorithm generates rich information on other entries of the relation table. Since the image of $\omega_{1}: \mathcal{S}^{(1)} \rightarrow \mathbb{R}$ is generically chosen, we assume without loss that there are exactly $n+1$ active constraints at any stage of the pivoting process, say,

$$
\begin{aligned}
\alpha_{0}= & \left\langle\hat{\mathbf{a}}_{1}^{(1)}, \hat{\alpha}\right\rangle, \\
\alpha_{0}= & \left\langle\hat{\mathbf{a}}_{l_{1}}^{(1)}, \hat{\alpha}\right\rangle, \\
& \vdots \\
\alpha_{0}= & \left\langle\hat{\mathbf{a}}_{l_{n}}^{(1)}, \hat{\alpha}\right\rangle, \\
\alpha_{0}< & \left\langle\hat{\mathbf{a}}_{k}^{(1)}, \hat{\alpha}\right\rangle, \quad \forall k \in\left\{2,3, \ldots, s_{1}\right\} \backslash\left\{l_{1}, \ldots, l_{n}\right\},
\end{aligned}
$$

then $\left[\hat{\mathbf{a}}_{j_{1}}^{(1)}, \hat{\mathbf{a}}_{j_{2}}^{(1)}\right]=1$ for all $j_{1}, j_{2} \in\left\{1, l_{1}, \ldots, l_{n}\right\}$ with $j_{1}<j_{2}$. This important feature considerably reduces the number of One-Point tests needed for completely determining the entries of the relation table.

To determine the rest of the unknown entries in the first row of the table in (5) from left to right: for $\left[\hat{\mathbf{a}}_{1}^{(1)}, \hat{\mathbf{a}}_{j}^{(1)}\right]$ for $j>2$, apply the One-Point test on $\hat{\mathbf{a}}_{j}^{(1)}$, or solve the LP problem

$$
\begin{aligned}
& \text { Minimize }\left\langle\hat{\mathbf{a}}_{j}^{(1)}, \hat{\alpha}\right\rangle-\alpha_{0} \\
& \quad \alpha_{0}=\left\langle\hat{\mathbf{a}}_{1}^{(1)}, \hat{\alpha}\right\rangle, \\
& \alpha_{0} \leq\left\langle\hat{\mathbf{a}}_{l}^{(1)}, \hat{\alpha}\right\rangle, \quad \forall l \in\left\{2, \ldots, s_{1}\right\},
\end{aligned}
$$

and for $\left[\hat{\mathbf{a}}_{1}^{(1)}, \hat{\mathbf{a}}_{j}^{(i)}\right]$ for $i>1, j \in\left\{1, \ldots, s_{i}\right\}$, solve the LP problem

$$
\begin{aligned}
& \operatorname{Minimize}\left\langle\hat{\mathbf{a}}_{j}^{(i)}, \hat{\alpha}\right\rangle-\alpha_{0} \\
& \left\langle\hat{\mathbf{a}}_{1}^{(1)}, \hat{\alpha}\right\rangle \leq\left\langle\hat{\mathbf{a}}_{l}^{(1)}, \hat{\alpha}\right\rangle, \quad \forall l \in\left\{2, \ldots, s_{1}\right\}, \\
& \alpha_{0} \leq\left\langle\hat{\mathbf{a}}_{m}^{(i)}, \hat{\alpha}\right\rangle, \quad \forall m \in\left\{1,2, \ldots, s_{i}\right\} .
\end{aligned}
$$

We let $\left[\hat{\mathbf{a}}_{1}^{(1)}, \hat{\mathbf{a}}_{j}^{(1)}\right]=1$, or $\left[\hat{\mathbf{a}}_{1}^{(1)}, \hat{\mathbf{a}}_{j}^{(i)}\right]=1$ if the corresponding optimal value is zero. They are zero otherwise.

Feasible points of the above LP problems are always available, there is no need to solve the sometimes costly Phase I problem here. Because when we determine $\left[\hat{\mathbf{a}}_{1}^{(1)}, \hat{\mathbf{a}}_{2}^{(1)}\right]$, there exists $\hat{\alpha}=(\alpha, 1)$ for which

$$
\left\langle\hat{\mathbf{a}}_{1}^{(1)}, \hat{\alpha}\right\rangle \leq\left\langle\hat{\mathbf{a}}_{l}^{(1)}, \hat{\alpha}\right\rangle, \quad \forall l \in\left\{2, \ldots, s_{1}\right\} .
$$


This $\hat{\alpha}$ together with $\alpha_{0}=\left\langle\hat{\mathbf{a}}_{1}^{(1)}, \hat{\alpha}\right\rangle$ for (9) or

$$
\alpha_{0}=\min \left\{\left\langle\hat{\mathbf{a}}_{m}^{(i)}, \hat{\alpha}\right\rangle \mid m=1, \ldots, s_{i}\right\}
$$

for (10) provides feasible points for the constraints of the respective LP problems.

A key observation here, and it will appear in all the LP problems below, is the possible removal of a substantial number of constraints in both (9) and (10). For instance, if we have known $\left[\hat{\mathbf{a}}_{1}^{(1)}, \hat{\mathbf{a}}_{\mu}^{(l)}\right]=0$ for certain $\mu \in\left\{1, \ldots, s_{l}\right\}$ and $l \in\{1, i\}$ before solving the LP problems in (9) or (10), then its corresponding constraint

$$
\left\langle\hat{\mathbf{a}}_{1}^{(1)}, \hat{\alpha}\right\rangle \leq\left\langle\hat{\mathbf{a}}_{\mu}^{(1)}, \hat{\alpha}\right\rangle \quad \text { if } \quad l=1 \quad \text { and } \quad i>1 \quad \text { or } \quad \alpha_{0} \leq\left\langle\hat{\mathbf{a}}_{\mu}^{(l)}, \hat{\alpha}\right\rangle \quad \text { otherwise }
$$

should be removed, because this constraint will never become active (otherwise, $\left[\hat{\mathbf{a}}_{1}^{(1)}\right.$, $\left.\hat{\mathbf{a}}_{\mu}^{(l)}\right]=1$ ) during the process of solving the LP problem. The removal of extraneous constraints of this sort, absent in the $\mathrm{Li}-\mathrm{Li}$ algorithm in [11], cumulatively yields a considerable reduction in the amount of computation in our algorithm, making it much superior to the existing ones even when applied to fully mixed systems without any special structures in the supports. In the following, we state the essence of this important observation as a proposition.

Proposition 1. In solving LP problem

$$
\begin{gathered}
\operatorname{Minimize}\langle\mathbf{f}, \mathbf{z}\rangle \\
\left\langle\mathbf{c}_{i}, \mathbf{z}\right\rangle \leq b_{i}, \quad i=1, \ldots, m,
\end{gathered}
$$

if it is known a priori that a certain constraint $\left\langle\mathbf{c}_{j}, \mathbf{z}\right\rangle \leq b_{j}$ will never become active during the process, then the solution remains invariant for the same LP problem without this constraint.

Now, consider the general row

$$
\hat{\mathbf{a}}_{\mu}^{(v)}: \overbrace{\left[\hat{\mathbf{a}}_{\mu}^{(v)}, \hat{\mathbf{a}}_{\mu+1}^{(v)}\right], \ldots,\left[\hat{\mathbf{a}}_{\mu}^{(v)}, \hat{\mathbf{a}}_{s_{v}}^{(v)}\right]}^{\mathrm{T}(v, v)}, \ldots, \overbrace{\left[\hat{\mathbf{a}}_{\mu}^{(v)}, \hat{\mathbf{a}}_{1}^{(r)}\right], \ldots,\left[\hat{\mathbf{a}}_{\mu}^{(v)}, \hat{\mathbf{a}}_{s_{r}}^{(r)}\right]}^{\mathrm{T}(v, r)}
$$

on the relation table, assuming all the entries in the previous rows have all been determined. As above, we determine $\left[\hat{\mathbf{a}}_{\mu}^{(v)}, \hat{\mathbf{a}}_{\mu+1}^{(v)}\right]$ in accordance with the optimal value of the LP problem

$$
\begin{aligned}
& \operatorname{Minimize}\left\langle\hat{\mathbf{a}}_{\mu+1}^{(v)}, \hat{\alpha}\right\rangle-\alpha_{0} \\
& \quad \alpha_{0}=\left\langle\hat{\mathbf{a}}_{\mu}^{(v)}, \hat{\alpha}\right\rangle, \\
& \quad \alpha_{0} \leq\left\langle\hat{\mathbf{a}}_{l}^{(v)}, \hat{\alpha}\right\rangle, \quad \forall l \in\left\{1, \ldots, s_{\nu}\right\} \backslash\{\mu\},
\end{aligned}
$$

i.e., $\left[\hat{\mathbf{a}}_{\mu}^{(v)}, \hat{\mathbf{a}}_{\mu+1}^{(v)}\right]=1$ if the optimal value is zero, $\left[\hat{\mathbf{a}}_{\mu}^{(v)}, \hat{\mathbf{a}}_{\mu+1}^{(v)}\right]=0$ otherwise.

If there exists $l_{0}<\mu$ where $\left[\hat{\mathbf{a}}_{l_{0}}^{(v)}, \hat{\mathbf{a}}_{\mu}^{(v)}\right]$ is known to be positive, then the functional $\hat{\alpha}=(\alpha, 1) \in\left(\mathbb{R}^{n+1}\right)^{\vee}$ is available for which

$$
\left\langle\hat{\mathbf{a}}_{l_{0}}^{(v)}, \hat{\alpha}\right\rangle=\left\langle\hat{\mathbf{a}}_{\mu}^{(v)}, \hat{\alpha}\right\rangle \leq\left\langle\hat{\mathbf{a}}_{l}^{(v)}, \hat{\alpha}\right\rangle, \quad \forall l \in\left\{1, \ldots, s_{\nu}\right\} \backslash\left\{l_{0}, \mu\right\} .
$$


This $\hat{\alpha}$ decides a feasible point of the problem. Solving the Phase I problem for a feasible point of this LP problem is only needed otherwise. Furthermore, for those points $\hat{\mathbf{a}}_{m}^{(v)}$ with $m<\mu$ for which we already know their negative relations with $\hat{\mathbf{a}}_{\mu}^{(v)}$, i.e., $\left[\hat{\mathbf{a}}_{m}^{(v)}, \hat{\mathbf{a}}_{\mu}^{(\nu)}\right]=0$, the corresponding constraints

$$
\alpha_{0} \leq\left\langle\hat{\mathbf{a}}_{m}^{(v)}, \hat{\alpha}\right\rangle
$$

will never become active. By Proposition 1, those constraints should be removed before solving the problem.

Similarly, to determine the rest of the entries in the row in (12): for the unknown entries $\left[\hat{\mathbf{a}}_{\mu}^{(v)}, \hat{\mathbf{a}}_{j}^{(\nu)}\right]$ for $j>\mu+1$, we solve the LP problem

$$
\begin{aligned}
& \operatorname{Minimize}\left\langle\hat{\mathbf{a}}_{j}^{(v)}, \hat{\alpha}\right\rangle-\alpha_{0} \\
& \quad \alpha_{0}=\left\langle\hat{\mathbf{a}}_{\mu}^{(\nu)}, \hat{\alpha}\right\rangle, \\
& \alpha_{0} \leq\left\langle\hat{\mathbf{a}}_{l}^{(\nu)}, \hat{\alpha}\right\rangle, \quad \forall l \in\left\{1, \ldots, s_{\nu}\right\} \backslash\{\mu\},
\end{aligned}
$$

and for $\left[\hat{\mathbf{a}}_{\mu}^{(v)}, \hat{\mathbf{a}}_{j}^{(i)}\right]$ for $j \in\left\{1, \ldots, s_{i}\right\}$ and $v<i \leq r$, solve the LP problem

$$
\begin{aligned}
\operatorname{Minimize} & \left\langle\hat{\mathbf{a}}_{j}^{(i)}, \hat{\alpha}\right\rangle-\alpha_{0} \\
\left\langle\hat{\mathbf{a}}_{\mu}^{(v)}, \hat{\alpha}\right\rangle & \leq\left\langle\hat{\mathbf{a}}_{l}^{(v)}, \hat{\alpha}\right\rangle, \quad \\
\alpha_{0} & \leq\left\langle\hat{\mathbf{a}}_{m}^{(i)}, \hat{\alpha}\right\rangle, \quad \forall m \in\left\{1, \ldots, s_{1}\right\} \backslash\{\mu\},
\end{aligned}
$$

Feasible points of those LP problems are always available with no need to solve the Phase I problem, because when we determine $\left[\hat{\mathbf{a}}_{\mu}^{(\nu)}, \hat{\mathbf{a}}_{\mu+1}^{(\nu)}\right]$, the existing $\hat{\alpha}=(\alpha, 1) \in$ $\left(\mathbb{R}^{n+1}\right)^{\vee}$ which satisfies

$$
\left\langle\hat{\mathbf{a}}_{\mu}^{(\nu)}, \hat{\alpha}\right\rangle \leq\left\langle\hat{\mathbf{a}}_{l}^{(\nu)}, \hat{\alpha}\right\rangle, \quad \forall l \in\left\{1, \ldots, s_{\nu}\right\} \backslash\{\mu\},
$$

provides a feasible point for the respective LP problem with a proper value of $\alpha_{0}$.

Again, by Proposition 1, before solving the LP problems, we remove the constraints corresponding to those points $\hat{\mathbf{a}}_{m}^{(l)}$ for $l \in\{v, v+1, \ldots, r\}$ whose relations with $\hat{\mathbf{a}}_{\mu}^{(v)}$ are known to be negative, that is, we have known either $\left[\hat{\mathbf{a}}_{\mu}^{(v)}, \hat{\mathbf{a}}_{m}^{(i)}\right]=0$ for $l=i>v$, or $\left[\hat{\mathbf{a}}_{\mu}^{(v)}, \hat{\mathbf{a}}_{m}^{(\nu)}\right]=0$ for $\mu<m$ or $\left[\hat{\mathbf{a}}_{m}^{(\nu)}, \hat{\mathbf{a}}_{\mu}^{(\nu)}\right]=0$ for $m<\mu$.

When we solve the LP problem in (15) by the simplex method, information generated by the pivoting process on other unknown entries of the table becomes particularly fruitful. We assume without loss of generality that there are exactly $n+1$ active constraints 
at any stage of the pivoting process, say

$$
\begin{aligned}
\left\langle\hat{\mathbf{a}}_{\mu}^{(v)}, \hat{\alpha}\right\rangle & =\left\langle\hat{\mathbf{a}}_{l_{1}}^{(v)}, \hat{\alpha}\right\rangle, \\
& \vdots \\
\left\langle\hat{\mathbf{a}}_{\mu}^{(v)}, \hat{\alpha}\right\rangle & =\left\langle\hat{\mathbf{a}}_{l_{s}}^{(v)}, \hat{\alpha}\right\rangle,
\end{aligned}
$$

and

$$
\begin{aligned}
\alpha_{0} & =\left\langle\hat{\mathbf{a}}_{m_{1}}^{(i)}, \hat{\alpha}\right\rangle, \\
& \vdots \\
\alpha_{0} & =\left\langle\hat{\mathbf{a}}_{m_{t}}^{(i)}, \hat{\alpha}\right\rangle,
\end{aligned}
$$

where $s+t=n+1$. Then for $l^{\prime}, l^{\prime \prime} \in\left\{\mu, l_{1}, \ldots, l_{s}\right\}$ with $l^{\prime}<l^{\prime \prime}$ and $m^{\prime}, m^{\prime \prime} \in$ $\left\{m_{1}, \ldots, m_{t}\right\}$ with $m^{\prime}<m^{\prime \prime}$, we have $\left[\hat{\mathbf{a}}_{l^{\prime}}^{(v)}, \hat{\mathbf{a}}_{l^{\prime \prime}}^{(v)}\right]=1$ and $\left[\hat{\mathbf{a}}_{m^{\prime}}^{(i)}, \hat{\mathbf{a}}_{m^{\prime \prime}}^{(i)}\right]=1$, and, for $l_{0} \in\left\{\mu, l_{1}, \ldots, l_{s}\right\}$ and $m_{0} \in\left\{m_{1}, \ldots, m_{t}\right\},\left[\hat{\mathbf{a}}_{l_{0}}^{(\nu)}, \hat{\mathbf{a}}_{m_{0}}^{(i)}\right]=1$.

\section{Level- $\xi$ Subfaces and Their Extensions}

For $1 \leq \xi \leq r$ and $\hat{F}_{i} \subset \hat{S}^{(i)}$ with $\operatorname{dim}\left(\hat{F}_{i}\right)=d_{i}$ for $i=1, \ldots, \xi,\left(\hat{F}_{1}, \ldots, \hat{F}_{\xi}\right)$ is called a level- $\xi$ subface of $\hat{S}=\left(\hat{S}^{(1)}, \ldots, \hat{S}^{(r)}\right)$ of type $\left(d_{1}, \ldots, d_{\xi}\right)$ if there exists $\hat{\alpha}=(\alpha, 1) \in \mathbb{R}^{n+1}$ such that for each $i=1, \ldots, \xi$,

$$
\left\langle\hat{\mathbf{a}}^{(i)}, \hat{\alpha}\right\rangle=\left\langle\hat{\mathbf{a}}^{(i)^{\prime}}, \hat{\alpha}\right\rangle, \quad \forall \hat{\mathbf{a}}^{(i)}, \hat{\mathbf{a}}^{(i)^{\prime}} \in \hat{F}_{i}
$$

and

$$
\left\langle\hat{\mathbf{a}}^{(i)}, \hat{\alpha}\right\rangle \leq\left\langle\hat{\mathbf{a}}^{(i)^{\prime \prime}}, \hat{\alpha}\right\rangle, \quad \forall \hat{\mathbf{a}}^{(i)} \in \hat{F}_{i} \quad \text { and } \quad \hat{\mathbf{a}}^{(i)^{\prime \prime}} \in \hat{S}^{(i)} \backslash \hat{F}_{i} .
$$

Equivalently, $\hat{F}_{i}$ is a lower face of $\hat{S}^{(i)}$ of dimension $d_{i}$ for each $i=1, \ldots, \xi$. A level- $\xi$ subface $\left(\hat{F}_{1}, \ldots, \hat{F}_{\xi}\right)$ of type $\left(k_{1}, \ldots, k_{\xi}\right)$ is said to be extendible if there is a lower face $\hat{F}_{\xi+1}$ of $\hat{S}^{(\xi+1)}$ which makes $\left(\hat{F}_{1}, \ldots, \hat{F}_{\xi}, \hat{F}_{\xi+1}\right)$ a level- $(\xi+1)$ subface. It is nonextendible otherwise.

A level-r subface $\left(\hat{F}_{1}, \ldots, \hat{F}_{r}\right)$ of $\hat{S}=\left(\hat{S}^{(1)}, \ldots, \hat{S}^{(r)}\right)$ of type $\left(k_{1}, \ldots, k_{r}\right)$ is a lower facet of $\hat{S}$ of type $\left(k_{1}, \ldots, k_{r}\right)$ when

$$
\begin{aligned}
\operatorname{dim}\left(\hat{F}_{1}+\cdots+\hat{F}_{r}\right) & =\operatorname{dim}\left(\hat{F}_{1}\right)+\cdots+\operatorname{dim}\left(\hat{F}_{r}\right) \\
& =k_{1}+\cdots+k_{r}=n .
\end{aligned}
$$

In such a case, $\left(F_{1}, \ldots, F_{r}\right)$ becomes a cell of type $\left(k_{1}, \ldots, k_{r}\right)$ in $S_{\omega}$. To find all such lower facets for calculating the mixed volume of a semi-mixed system $P(\mathbf{x})$ of type $\left(k_{1}, \ldots, k_{r}\right)$ with support $\mathcal{S}=\left(\mathcal{S}^{(1)}, \ldots, \mathcal{S}^{(r)}\right)$, we find all level-1 subfaces of $\hat{\mathcal{S}}=\left(\hat{\mathcal{S}}^{(1)}, \ldots, \hat{\mathcal{S}}^{(r)}\right)$ of type $\left(k_{1}\right)$ in the first place, followed by extending each such subface step by step to a level-r subface of $\hat{\mathcal{S}}$ of type $\left(k_{1}, \ldots, k_{r}\right)$. 
4.1. Level-1 Subfaces of $\hat{\mathcal{S}}=\left(\hat{\mathcal{S}}^{(1)}, \ldots, \hat{\mathcal{S}}^{(r)}\right)$

Apparently, level-1 subfaces of $\hat{\mathcal{S}}=\left(\hat{\mathcal{S}}^{(1)}, \ldots, \hat{\mathcal{S}}^{(r)}\right)$ of type $\left(k_{1}\right)$ are faces of dimension $k_{1}$ in the lower hull of $\hat{\mathcal{S}}^{(1)}=\left\{\hat{\mathbf{a}}_{1}^{(1)}, \ldots, \hat{\mathbf{a}}_{s_{1}}^{(1)}\right\}$, they are faces of dimension $k_{1}$ of $\hat{\mathcal{S}}^{(1)}$ having an inner normal of type $\hat{\alpha}=(\alpha, 1) \in\left(\mathbb{R}^{n+1}\right)^{\vee}$. When $k_{1}=1$, such subfaces are the pairs of points $\left\{\mathbf{a}_{l_{0}}^{(1)}, \mathbf{a}_{l_{1}}^{(1)}\right\}$ on the relation table $\mathrm{T}(1,1)$ with $\left[\mathbf{a}_{l_{0}}^{(1)}, \mathbf{a}_{l_{1}}^{(1)}\right]=1$, $1 \leq l_{0}<l_{1} \leq s_{1}$. So only the case $k_{1}>1$ is discussed here. We will attain all those faces by extending each lower face of $\hat{\mathcal{S}}^{(1)}$ of dimension one, or a lower $e$ dge of $\hat{\mathcal{S}}^{(1)}$, step by step, to a lower face of $\hat{\mathcal{S}}^{(1)}$ of dimension $k_{1}$. More precisely, for lower edge $\left\{\hat{\mathbf{a}}_{l_{0}}^{(1)}, \hat{\mathbf{a}}_{l_{1}}^{(1)}\right\}$ of $\hat{\mathcal{S}}^{(1)}$ with $l_{0}<l_{1}$, we look for all possible points $\hat{\mathbf{a}}_{l}^{(1)}$ in $\hat{\mathcal{S}}^{(1)}$ with $l>l_{1}$ for which $\left\{\hat{\mathbf{a}}_{l_{0}}^{(1)}, \hat{\mathbf{a}}_{l_{1}}^{(1)}, \hat{\mathbf{a}}_{l}^{(1)}\right\}$ is a lower face of $\hat{\mathcal{S}}^{(1)}$ of dimension two. Inductively, for a known lower face $\left\{\hat{\mathbf{a}}_{l_{0}}^{(1)}, \hat{\mathbf{a}}_{l_{1}}^{(1)}, \ldots, \hat{\mathbf{a}}_{l_{j}}^{(1)}\right\}$ of $\hat{\mathcal{S}}^{(1)}$ of dimension $j$ with $j<k_{1}$ and $l_{0}<l_{1}<\cdots<l_{j}$, we look for all possible points $\hat{\mathbf{a}}_{l}^{(1)}$ in $\hat{\mathcal{S}}^{(1)}$ with $l>l_{j}$ for which $\left\{\hat{\mathbf{a}}_{l_{0}}^{(1)}, \hat{\mathbf{a}}_{l_{1}}^{(1)}, \ldots, \hat{\mathbf{a}}_{l_{j}}^{(1)}, \hat{\mathbf{a}}_{l}^{(1)}\right\}$ is a lower face of $\hat{\mathcal{S}}^{(1)}$ of dimension $j+1$. Lower face $\left\{\hat{\mathbf{a}}_{l_{0}}^{(1)}, \hat{\mathbf{a}}_{l_{1}}^{(1)}, \ldots, \hat{\mathbf{a}}_{l_{j}}^{(1)}\right\}$ is called extendible if such a point exists. This task of extension can be carried out systematically by employing the One-Point test successively.

We extend lower edges of $\hat{\mathcal{S}}^{(1)}$ one by one in the order from left to right and top to bottom of their corresponding entries on the relation table $\mathrm{T}(1,1)$.

For $\left[\hat{\mathbf{a}}_{l_{0}}^{(1)}, \hat{\mathbf{a}}_{l_{1}}^{(1)}\right]=1$ where $1 \leq l_{0}<l_{1}<s_{1}$, we first identify on table $\mathrm{T}(1,1)$ the set

$$
\mathcal{C}^{(1)}=\left\{l \mid 1 \leq l \leq s_{1}, \hat{\mathbf{a}}_{l}^{(1)} \text { has positive relations with both } \hat{\mathbf{a}}_{l_{0}}^{(1)} \text { and } \hat{\mathbf{a}}_{l_{1}}^{(1)}\right\},
$$

and let $\mathcal{T}^{(1)}$ be the elements in $\mathcal{C}^{(1)}$ which are bigger than $l_{1}$, i.e.,

$$
\mathcal{T}^{(1)}=\left\{l \mid l>l_{1},\left[\hat{\mathbf{a}}_{l_{0}}^{(1)}, \hat{\mathbf{a}}_{l}^{(1)}\right]=\left[\hat{\mathbf{a}}_{l_{1}}^{(1)}, \hat{\mathbf{a}}_{l}^{(1)}\right]=1\right\} .
$$

Clearly, the set

$$
\mathcal{P}^{(1)}=\left\{\hat{\mathbf{a}}_{l}^{(1)} \mid l \in \mathcal{T}^{(1)}\right\}
$$

contains all the possible points which may subsequently extend $\left\{\hat{\mathbf{a}}_{l_{0}}^{(1)}, \hat{\mathbf{a}}_{l_{1}}^{(1)}\right\}$ to a $k_{1^{-}}$ dimensional lower face $\left\{\hat{\mathbf{a}}_{l_{0}}^{(1)}, \ldots, \hat{\mathbf{a}}_{l_{1}}^{(1)}\right\}$ of $\hat{\mathcal{S}}^{(1)}$ with $l_{0}<l_{1}<\cdots<l_{k_{1}}$. Let $s$ be the number of points in $\mathcal{P}^{(1)}$. Obviously, if $s<k_{1}-1$, such a $k_{1}$-dimensional lower face does not exist, and the edge $\left\{\hat{\mathbf{a}}_{l_{0}}^{(1)}, \hat{\mathbf{a}}_{l_{1}}^{(1)}\right\}$ will eventually be nonextendible. In such a case, we switch our extension consideration to the next lower edge. When $s \geq k_{1}-1$, the extendibility of $\left\{\hat{\mathbf{a}}_{l_{0}}^{(1)}, \hat{\mathbf{a}}_{l_{1}}^{(1)}\right\}$ will be determined by examining the points in $\mathcal{P}^{(1)}$. Let

$$
\tau_{1}<\tau_{2}<\cdots<\tau_{s}
$$

be the elements in $\mathcal{T}^{(1)}$. To begin, we consider the LP problem

$$
\begin{gathered}
\text { Minimize }\left\langle\hat{\mathbf{a}}_{\tau_{1}}^{(1)}, \hat{\alpha}\right\rangle-\alpha_{0} \\
\alpha_{0}=\left\langle\hat{\mathbf{a}}_{l_{0}}^{(1)}, \hat{\alpha}\right\rangle=\left\langle\hat{\mathbf{a}}_{l_{1}}^{(1)}, \hat{\alpha}\right\rangle, \\
\alpha_{0} \leq\left\langle\hat{\mathbf{a}}_{k}^{(1)}, \hat{\alpha}\right\rangle, \quad \forall k \in \mathcal{C}^{(1)},
\end{gathered}
$$

in the variables $\hat{\alpha}=(\alpha, 1) \in\left(\mathbb{R}^{n+1}\right)^{\vee}$ and $\alpha_{0} \in \mathbb{R}$. This is another form of the OnePoint test, it is used to test if the first point $\hat{\mathbf{a}}_{\tau_{1}}^{(1)}$ of $\mathcal{P}^{(1)}$ along with $\left\{\hat{\mathbf{a}}_{l_{0}}^{(1)}, \hat{\mathbf{a}}_{l_{1}}^{(1)}\right\}$ constitute a 
two-dimensional lower face of $\hat{\mathcal{S}}^{(1)}$. In general, by the term One-Point test used below, we always refer to the testing of a given point's possibility to extend either a lower face or a level- $\xi$ subface.

A feasible point of this problem is available since $\left[\hat{\mathbf{a}}_{l_{0}}^{(1)}, \hat{\mathbf{a}}_{l_{1}}^{(1)}\right]=1$ implies the existence of $\hat{\alpha} \in\left(\mathbb{R}^{n+1}\right)^{\vee}$ for which

$$
\left\langle\hat{\mathbf{a}}_{l_{0}}^{(1)}, \hat{\alpha}\right\rangle=\left\langle\hat{\mathbf{a}}_{l_{1}}^{(1)}, \hat{\alpha}\right\rangle
$$

and

$$
\left\langle\hat{\mathbf{a}}_{l_{0}}^{(1)}, \hat{\alpha}\right\rangle \leq\left\langle\hat{\mathbf{a}}_{k}^{(1)}, \hat{\alpha}\right\rangle, \quad \forall k \in\left\{1, \ldots, s_{1}\right\} \backslash\left\{l_{0}, l_{1}\right\} .
$$

This $\hat{\alpha}$ along with

$$
\alpha_{0}=\min _{k \in \mathcal{C}^{(1)}}\left\langle\hat{\mathbf{a}}_{k}^{(1)}, \hat{\alpha}\right\rangle
$$

yield a feasible point of the constraints in (16). Clearly, the zero optimal value of this LP problem makes $\left\{\hat{\mathbf{a}}_{l_{0}}^{(1)}, \hat{\mathbf{a}}_{l_{1}}^{(1)}, \hat{\mathbf{a}}_{\tau_{1}}^{(1)}\right\}$ a lower face of $\hat{\mathcal{S}}^{(1)}$ of dimension two, and the point $\hat{\mathbf{a}}_{\tau_{1}}^{(1)}$ will be retained for further extension considerations. When the optimal value is not zero, $\hat{\mathbf{a}}_{\tau_{1}}^{(1)}$ fails to extend $\left\{\hat{\mathbf{a}}_{l_{0}}^{(1)}, \hat{\mathbf{a}}_{l_{1}}^{(1)}\right\}$ and will be deleted.

Again, the pivoting process in solving the LP problem in (16) by the simplex method provides abundant information on the extendibility of $\left\{\hat{\mathbf{a}}_{l_{0}}^{(1)}, \hat{\mathbf{a}}_{l_{1}}^{(1)}\right\}$ by other points in $\mathcal{P}^{(1)}$. For instance, at any stage of the pivoting process, when the set of active constraints contains

$$
\alpha_{0}=\left\langle\hat{\mathbf{a}}_{l}^{(1)}, \hat{\alpha}\right\rangle
$$

for any $l \in \mathcal{T}^{(1)} \backslash\left\{\tau_{1}\right\}$, then $\hat{\mathbf{a}}_{l}^{(1)}$ extends $\left\{\hat{\mathbf{a}}_{l_{0}}^{(1)}, \hat{\mathbf{a}}_{l_{1}}^{(1)}\right\}$ and can be omitted from the list of further testings.

When we apply this One-Point test on the next point $\hat{\mathbf{a}}_{\tau}^{(1)}$ in $\mathcal{P}^{(1)}$ whose status in extending $\left\{\hat{\mathbf{a}}_{l_{0}}^{(1)}, \hat{\mathbf{a}}_{l_{1}}^{(1)}\right\}$ is undetermined, or solve the LP problem

$$
\begin{gathered}
\text { Minimize }\left\langle\hat{\mathbf{a}}_{\tau}^{(1)}, \hat{\alpha}\right\rangle-\alpha_{0} \\
\alpha_{0}=\left\langle\hat{\mathbf{a}}_{l_{0}}^{(1)}, \hat{\alpha}\right\rangle=\left\langle\hat{\mathbf{a}}_{l_{1}}^{(1)}, \hat{\alpha}\right\rangle, \\
\alpha_{0} \leq\left\langle\hat{\mathbf{a}}_{k}^{(1)}, \hat{\alpha}\right\rangle, \quad \forall k \in \mathcal{C}^{(1)},
\end{gathered}
$$

as in (16), those constraints

$$
\alpha_{0} \leq\left\langle\hat{\mathbf{a}}_{l}^{(1)}, \hat{\alpha}\right\rangle
$$

corresponding to points $\hat{\mathbf{a}}_{l}^{(1)}$ in $\mathcal{P}^{(1)}$ which fail to extend $\left\{\hat{\mathbf{a}}_{l_{0}}^{(1)}, \hat{\mathbf{a}}_{l_{1}}^{(1)}\right\}$, are clearly extraneous. By Proposition 1, they should be removed before we solve the LP problem.

When the testing on points of $\mathcal{P}^{(1)}$ is completed, we have extended $\left\{\hat{\mathbf{a}}_{l_{0}}^{(1)}, \hat{\mathbf{a}}_{l_{1}}^{(1)}\right\}$ to all possible two-dimensional lower faces. Let

$$
\mathcal{E}^{(1)}=\left\{\hat{\mathbf{a}}_{l}^{(1)} \in \mathcal{P}^{(1)} \mid\left\{\hat{\mathbf{a}}_{l_{0}}^{(1)}, \hat{\mathbf{a}}_{l_{1}}^{(1)}, \hat{\mathbf{a}}_{l}^{(1)}\right\} \text { is a two-dimensional lower face }\right\},
$$

and let $\bar{s}$ be the number of points in $\mathcal{E}^{(1)}$. As before, if $\bar{s}<k_{1}-1$, the edge $\left\{\hat{\mathbf{a}}_{l_{0}}^{(1)}, \hat{\mathbf{a}}_{l_{1}}^{(1)}\right\}$ will eventually be nonextendible and the extension attempt on $\left\{\hat{\mathbf{a}}_{l_{0}}^{(1)}, \hat{\mathbf{a}}_{l_{1}}^{(1)}\right\}$ will be terminated. 
When $\bar{s} \geq k_{1}-1$, the extendibility of $\left\{\hat{\mathbf{a}}_{l_{0}}^{(1)}, \hat{\mathbf{a}}_{l_{1}}^{(1)}, \hat{\mathbf{a}}_{l}^{(1)}\right\}$ for each $\hat{\mathbf{a}}_{l}^{(1)} \in \mathcal{E}^{(1)}$ will continue to be tested. When we consider the extendibility of $\left\{\hat{\mathbf{a}}_{l_{0}}^{(1)}, \hat{\mathbf{a}}_{l_{1}}^{(1)}, \hat{\mathbf{a}}_{l_{2}}^{(1)}\right\}$ for $\hat{\mathbf{a}}_{l_{2}}^{(1)} \in \mathcal{E}^{(1)}$, only those points $\hat{\mathbf{a}}_{l}^{(1)}$ in $\mathcal{E}^{(1)}$ with $l>l_{2}$ need to be examined, and those constraints corresponding to points $\hat{\mathbf{a}}_{l}^{(1)}$ with $\left[\hat{\mathbf{a}}_{l_{2}}^{(1)}, \hat{\mathbf{a}}_{l}^{(1)}\right]=0$ for $l>l_{2}$ or $\left[\hat{\mathbf{a}}_{l}^{(1)}, \hat{\mathbf{a}}_{l_{2}}^{(1)}\right]=0$ for $l<l_{2}$ as well as the constraints corresponding to the points in $\mathcal{P}^{(1)} \backslash \mathcal{E}^{(1)}$ should be removed.

The process described above may be repeated in the same fashion when we attempt to extend a $j$-dimensional lower face $\left\{\hat{\mathbf{a}}_{l_{0}}^{(1)}, \ldots, \hat{\mathbf{a}}_{l_{j}}^{(1)}\right\}$ for $j<k_{1}$ to $(j+1)$-dimensional lower faces. In the end, all $k_{1}$-dimensional lower faces $\left\{\hat{\mathbf{a}}_{l_{0}}^{(1)}, \hat{\mathbf{a}}_{l_{1}}^{(1)} \ldots, \hat{\mathbf{a}}_{l_{k_{1}}}^{(1)}\right\}$ of $\hat{\mathcal{S}}^{(1)}$ with $l_{0}<l_{1}<\cdots<l_{k_{1}}$ can be found if they exist.

\subsection{The Extension of Level- $\xi$ Subfaces}

Let $\hat{E}_{\xi}=\left(\hat{F}_{1}, \ldots, \hat{F}_{\xi}\right)$ be a level- $\xi$ subface of $\hat{\mathcal{S}}=\left(\hat{\mathcal{S}}^{(1)}, \ldots, \hat{\mathcal{S}}^{(r)}\right)$ of type $\left(k_{1}, \ldots, k_{\xi}\right)$ with $\xi<r$ where $\hat{F}_{i} \subseteq \hat{\mathcal{S}}^{(i)}=\left\{\hat{\mathbf{a}}_{1}^{(i)}, \ldots, \hat{\mathbf{a}}_{s_{i}}^{(i)}\right\}$ for each $i=1, \ldots, \xi$. To continue the extension of $\hat{E}_{\xi}$, we look for lower faces $\left\{\hat{F}_{\xi+1}\right\}$ of $\hat{\mathcal{S}}^{(\xi+1)}=\left\{\hat{\mathbf{a}}_{1}^{(\xi+1)}, \ldots, \hat{\mathbf{a}}_{\xi_{\xi+1}}^{(\xi+1)}\right\}$ of dimension $k_{\xi+1}$ so that $\hat{E}_{\xi+1}=\left(\hat{F}_{1}, \ldots, \hat{F}_{\xi+1}\right)$ is a level- $(\xi+1)$ subface of $\hat{\mathcal{S}}$ of type $\left(k_{1}, \ldots, k_{\xi+1}\right)$. To find all such lower faces, we first find all the vertices $\hat{a}_{l}^{(\xi+1)}$ in the lower hull of $\hat{S}^{(\xi+1)}$ for which $\left(\hat{F}_{1}, \ldots, \hat{F}_{\xi},\left\{\hat{a}_{l}^{(\xi+1)}\right\}\right)$ is a level- $(\xi+1)$ subface of $\hat{S}$ of type $\left(k_{1}, \ldots, k_{\xi}, 0\right)$, followed by extending each such vertex of $\hat{S}^{(\xi+1)}$ to lower faces $\hat{F}_{\xi+1}^{j}$ of $\hat{S}^{(\xi+1)}$ of dimension $j$ for $j=1, \ldots, k_{\xi+1}$ consecutively, where for each $j, \hat{F}_{\xi+1}^{j} \subset \hat{F}_{\xi+1}^{j+1}$ and $\left(\hat{F}_{1}, \ldots, \hat{F}_{\xi}, \hat{F}_{\xi+1}^{j}\right)$ is a level- $(\xi+1)$ subface of $\hat{S}$ of type $\left(k_{1}, \ldots, k_{\xi}, j\right)$.

For each $i=1, \ldots, \xi$, since $\operatorname{dim}\left(\hat{F}_{i}\right)=k_{i}$, let

$$
\hat{F}_{i}=\left\{\hat{\mathbf{a}}_{l_{0}}^{(i)}, \ldots, \hat{\mathbf{a}}_{l_{k_{i}}}^{(i)}\right\}
$$

To extend $\hat{E}_{\xi}$, we begin by collecting on table $\mathrm{T}(i, \xi+1), i=1, \ldots, \xi$, all the points $\hat{\mathbf{a}}_{l}^{(\xi+1)}$ in $\hat{\mathcal{S}}^{(\xi+1)}$ where $\left[\hat{\mathbf{a}}_{l_{j}}^{(i)}, \hat{\mathbf{a}}_{l}^{(\xi+1)}\right]=1$ for all $j=0, \ldots, k_{i}$ and $i=1, \ldots, \xi$, and denote the set of all those points by $\mathcal{P}^{(\xi+1)}$. This set clearly contains all the vertex points of any lower face of $\hat{\mathcal{S}}^{(\xi+1)}$ of dimension $k_{\xi+1}$ that extends $\hat{E}_{\xi}$. Thus, $\hat{E}_{\xi}$ is nonextendible if the number of points in $\mathcal{P}^{(\xi+1)}$, denoted by $s^{\prime}$, is less than $k_{\xi+1}+1$. When $s^{\prime} \geq k_{\xi+1}+1$, let

$$
\tau_{1}^{\prime}<\tau_{2}^{\prime}<\cdots<\tau_{s^{\prime}}^{\prime}
$$

be the indices of the points in $\mathcal{P}^{(\xi+1)}$. In this order, we start to examine $\hat{\mathbf{a}}_{\tau_{1}^{\prime}}^{(\xi+1)}$ for its possibility to extend $\hat{E}_{\xi}$ by considering the One-Point test on $\hat{\mathbf{a}}_{\tau_{1}^{\prime}}^{(\xi+1)}$ :

$$
\begin{aligned}
& \operatorname{Minimize}\left\langle\hat{\mathbf{a}}_{\tau_{1}^{\prime}}^{(\xi+1)}, \hat{\alpha}\right\rangle-\alpha_{0}
\end{aligned}
$$

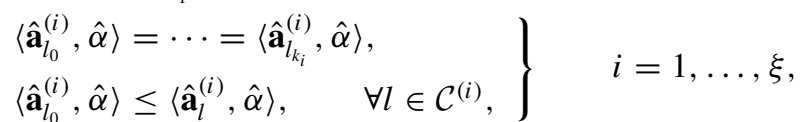

$$
\begin{aligned}
& \alpha_{0} \leq\left\langle\hat{\mathbf{a}}_{k}^{(\xi+1)}, \hat{\alpha}\right\rangle, \quad \forall k \in \mathcal{C}^{(\xi+1)},
\end{aligned}
$$


in the variables $\hat{\alpha}=(\alpha, 1) \in\left(\mathbb{R}^{n+1}\right)^{\vee}$ and $\alpha_{0} \in \mathbb{R}$, where, for $i=1, \ldots, \xi, \mathcal{C}^{(i)}$ is the set of indices of points $\hat{\mathbf{a}}_{l}^{(i)}$ in $\hat{\mathcal{S}}^{(i)}$ with $\left[\hat{\mathbf{a}}_{l_{j}}^{(i)}, \hat{\mathbf{a}}_{l}^{(i)}\right]=1$ for all $j=0, \ldots, k_{i}$, and $\mathcal{C}^{(\xi+1)}$ contains the indices of the points in $\mathcal{P}^{(\xi+1)}$.

We do not repeat here the details concerning the feasibility of the LP problem as well as the rich information generated by the pivoting process of the simplex algorithm on other points of $\mathcal{P}^{(\xi+1)}$ in extending $\hat{E}_{\xi}$. In short, feasible points of (18) are always available without having to solve the Phase I problem, and, most importantly, the amount of OnePoint tests one must apply can be considerably reduced by the information generated by the simplex pivoting. When the optimal value is zero, the point $\hat{\mathbf{a}}_{\tau_{1}^{\prime}}^{(\xi+1)}$ will be retained for further extension considerations, otherwise it would be deleted.

Before we solve the LP problem (18), a large number of constraints may be removed. In fact, when $\hat{E}_{\xi}=\left(\hat{F}_{1}, \ldots, \hat{F}_{\xi}\right)$ was constructed by step-by-step extensions, many constraints have been gradually removed along the route of the extension. One must update those remaining constraints when $\hat{E}_{\xi}$ is extended further. For instance, for $i=1, \ldots, \xi$, let $\mathcal{D}^{(i)}$ be the set of indices of points in $\hat{\mathcal{S}}^{(i)}$ whose corresponding constraints were used in the LP problems for testing the extendibility of the level- $\xi$ subface $\left(\hat{F}_{1}, \ldots, \hat{F}_{\xi} \backslash\left\{\hat{\mathbf{a}}_{l_{k \xi}}^{(\xi)}\right\}\right)$ of $\hat{\mathcal{S}}$ of type $\left(k_{1}, \ldots, k_{\xi-1}, k_{\xi}-1\right)$. Let

$$
\begin{aligned}
& \mathcal{C}_{0}^{(i)}=\left\{l \in \mathcal{D}^{(i)} \mid\left[\hat{\mathbf{a}}_{l}^{(i)}, \hat{\mathbf{a}}_{l_{\xi \xi}}^{(\xi)}\right]=1\right\} \quad \text { for } \quad i=1, \ldots, \xi-1, \\
& \mathcal{C}_{0}^{(\xi)}=\left\{l \in \mathcal{D}^{(\xi)} \mid\left[\hat{\mathbf{a}}_{l}^{(\xi)}, \hat{\mathbf{a}}_{l_{k_{\xi}}}^{(\xi)}\right]=1 \text { when } l<l_{k_{\xi}} \text { or }\left[\hat{\mathbf{a}}_{l_{k_{\xi}}}^{(\xi)}, \hat{\mathbf{a}}_{l}^{(\xi)}\right]=1 \text { when } l_{k_{\xi}}<l\right\} .
\end{aligned}
$$

Then the LP problem in (18) becomes

$$
\begin{gathered}
\operatorname{Minimize}\left\langle\hat{\mathbf{a}}_{\tau_{1}^{\prime}}^{(\xi+1)}, \hat{\alpha}\right\rangle-\alpha_{0} \\
\left.\begin{array}{rl}
\left\langle\hat{\mathbf{a}}_{l_{0}}^{(i)}, \hat{\alpha}\right\rangle & =\cdots=\left\langle\hat{\mathbf{a}}_{l_{k_{i}}}^{(i)}, \hat{\alpha}\right\rangle, \\
\left\langle\hat{\mathbf{a}}_{l_{0}}^{(i)}, \hat{\alpha}\right\rangle & \leq\left\langle\hat{\mathbf{a}}_{l}^{(i)}, \hat{\alpha}\right\rangle, \quad \forall l \in \mathcal{C}_{0}^{(i)},
\end{array}\right\} \quad i=1, \ldots, \xi, \\
\alpha_{0} \leq\left\langle\hat{\mathbf{a}}_{k}^{(\xi+1)}, \hat{\alpha}\right\rangle, \quad \forall k \in \mathcal{C}^{(\xi+1)}
\end{gathered}
$$

Now, when the One-Point test is applied to another point $\hat{\mathbf{a}}_{\tau_{j}^{\prime}}^{(\xi+1)}$ in $\mathcal{P}^{(\xi+1)}$ to determine its status in extending $\hat{E}_{\xi}$, we may solve the corresponding LP problem inheriting the constraint set in (19) with the possible removal of additional constraints.

When the examination on the points in $\mathcal{P}^{(\xi+1)}$ for the extension of $\hat{E}_{\xi}$ is completed, let $\mathcal{E}^{(\xi+1)}$ be the set of points in $\mathcal{P}^{(\xi+1)}$ which are capable of extending $\hat{E}_{\xi}$; namely, for each such point $\hat{\mathbf{a}}_{l}^{(\xi+1)},\left(\hat{F}_{1}, \ldots, \hat{F}_{\xi},\left\{\hat{\mathbf{a}}_{l}^{(\xi+1)}\right\}\right)$ is a level- $(\xi+1)$ subface of $\hat{\mathcal{S}}$ of type $\left(k_{1}, \ldots, k_{\xi}, 0\right)$. If the number of points in $\mathcal{E}^{(\xi+1)}$ is no less than $k_{\xi+1}+1$, let the indices of its points be

$$
\tilde{\tau}_{1}<\tilde{\tau}_{2}<\cdots<\tilde{\tau}_{\tilde{s}}
$$

and, in this order, we continue our attempt to extend

$$
\left(\hat{F}_{1}, \ldots, \hat{F}_{\xi},\left\{\hat{\mathbf{a}}_{\tilde{\tau}_{j}}^{(\xi+1)}\right\}\right)
$$


for $j=1, \ldots, \tilde{s}$, by examining points in $\left\{\hat{\mathbf{a}}_{\tilde{\tau}_{l}}^{(\xi+1)}\right\}_{l>j} \subset \mathcal{E}^{(\xi+1)}$. That is, for fixed $\hat{\mathbf{a}}_{\tilde{\tau}_{j}}^{(\xi+1)}$, and $l>j$, we solve the LP problem

$$
\begin{aligned}
& \text { Minimize }\left\langle\hat{\mathbf{a}}_{\tilde{\tau}_{l}}^{(\xi+1)}, \hat{\alpha}\right\rangle-\alpha_{0} \\
&\left\langle\hat{\mathbf{a}}_{l_{0}}^{(i)}, \hat{\alpha}\right\rangle=\cdots=\left\langle\hat{\mathbf{a}}_{l_{k_{i}}}^{(i)}, \hat{\alpha}\right\rangle, \quad \forall m \in \mathcal{C}_{1}^{(i)}, \\
&\left\langle\hat{\mathbf{a}}_{l_{0}}^{(i)}, \hat{\alpha}\right\rangle \leq\left\langle\hat{\mathbf{a}}_{m}^{(i)}, \hat{\alpha}\right\rangle, \quad i=1, \ldots, \xi, \\
& \alpha_{0}=\left\langle\hat{\mathbf{a}}_{\tau_{j}}^{(\xi+1)}, \hat{\alpha}\right\rangle, \\
& \alpha_{0} \leq\left\langle\hat{\mathbf{a}}_{m}^{(\xi+1)}, \hat{\alpha}\right\rangle, \quad \forall m \in \mathcal{C}_{1}^{(\xi+1)},
\end{aligned}
$$

in the variables $\hat{\alpha}=(\alpha, 1) \in\left(\mathbb{R}^{n+1}\right)^{\vee}$ and $\alpha_{0} \in \mathbb{R}$, where

$$
\mathcal{C}_{1}^{(i)}=\left\{m \mid m \in \mathcal{C}_{0}^{(i)} \text { and }\left[\hat{\mathbf{a}}_{m}^{(i)}, \hat{\mathbf{a}}_{\tilde{\tau}_{j}}^{(\xi+1)}\right]=1\right\}, \quad i=1, \ldots, \xi,
$$

and

$$
\mathcal{C}_{1}^{(\xi+1)}=\mathcal{C}^{(\xi+1)} \backslash\left\{m \mid m<l \text { and } \hat{\mathbf{a}}_{m}^{(\xi+1)} \text { fails to extend }\left(\hat{F}_{1}, \ldots, \hat{F}_{\xi},\left\{\hat{\mathbf{a}}_{\tilde{\tau}_{j}}^{(\xi+1)}\right\}\right)\right\},
$$

to determine if

$$
\left(\hat{F}_{1}, \ldots, \hat{F}_{\xi},\left\{\hat{\mathbf{a}}_{\tilde{\tau}_{j}}^{(\xi+1)}, \hat{\mathbf{a}}_{\tilde{\tau}_{l}}^{(\xi+1)}\right\}\right)
$$

forms a level- $(\xi+1)$ subface of $\hat{\mathcal{S}}$ of type $\left(k_{1}, \ldots, k_{\xi}, 1\right)$.

This procedure may be continued along the same line as we extend the lower faces of $\hat{\mathcal{S}}_{1}$ until all subfaces

$$
\hat{F}_{\xi+1}=\left\{\hat{\mathbf{a}}_{l_{0}}^{(\xi+1)}, \ldots, \hat{\mathbf{a}}_{s_{\xi}}^{(\xi+1)}\right\}
$$

of $\hat{\mathcal{S}}^{(\xi+1)}$ of dimension $k_{\xi+1}$ for which

$$
\hat{E}_{\xi+1}:=\left(\hat{F}_{1}, \ldots, \hat{F}_{\xi}, \hat{F}_{\xi+1}\right)
$$

are level- $(\xi+1)$ subfaces of $\hat{\mathcal{S}}$ of type $\left(k_{1}, \ldots, k_{\xi}, k_{\xi+1}\right)$ are obtained.

\section{Numerical Results}

As we mentioned in Section 2, the mixed volume of a semi-mixed polynomial system $P(\mathbf{x})=\left(p_{1}(\mathbf{x}), \ldots, p_{n}(\mathbf{x})\right)$ of type $\left(k_{1}, \ldots, k_{r}\right)$ with support $\mathcal{S}=\left(\mathcal{S}^{(1)}, \ldots, \mathcal{S}^{(r)}\right)$ equals the sum of the $n$-dimensional volumes of all mixed cells of type $\left(k_{1}, \ldots, k_{r}\right)$ in a fine mixed subdivision $S_{\omega}$ of $\mathcal{S}=\left(\mathcal{S}^{(1)}, \ldots, \mathcal{S}^{(r)}\right)$ induced by a generic lifting $\omega=\left(\omega_{1}, \ldots, \omega_{r}\right)$ on $\mathcal{S}$. More precisely, if

$$
C=\left(\left\{\mathbf{a}_{l_{0}}^{(1)}, \ldots, \mathbf{a}_{l_{k_{1}}}^{(1)}\right\}, \ldots,\left\{\mathbf{a}_{l_{0}}^{(r)}, \ldots, \mathbf{a}_{l_{k_{r}}}^{(r)}\right\}\right)
$$


represents such a mixed cell where $\left\{\mathbf{a}_{l_{0}}^{(i)}, \ldots, \mathbf{a}_{l_{k_{i}}}^{(i)}\right\} \subset \mathcal{S}^{(i)}$ for $i=1, \ldots, r$ and $k_{1}+\cdots+$ $k_{r}=n$, then

$$
\mathcal{M}(\mathcal{S})=\sum_{\mathcal{C}}\left|\operatorname{det}\left(\begin{array}{c}
\mathbf{a}_{l_{1}}^{(1)}-\mathbf{a}_{l_{0}}^{(1)} \\
\vdots \\
\mathbf{a}_{l_{k_{1}}}^{(1)}-\mathbf{a}_{l_{0}}^{(1)} \\
\vdots \\
\mathbf{a}_{l_{1}}^{(r)}-\mathbf{a}_{l_{0}}^{(r)} \\
\vdots \\
\mathbf{a}_{l_{k_{r}}}^{(r)}-\mathbf{a}_{l_{0}}^{(r)}
\end{array}\right)\right|
$$

Accordingly, when all mixed cells of $S_{\omega}$ of type $\left(k_{1}, \ldots, k_{r}\right)$ are available, the mixed volume of the system can be assembled with little extra computational effort.

Our algorithm for computing the mixed volume of a semi-mixed polynomial system this way has been successfully implemented and tested on a large variety of polynomial systems. The numerical results clearly demonstrate that a considerable speed up can be achieved when the special structure of the support of a semi-mixed polynomial system is taken into account. Even when applied to fully mixed systems with no special structure in the support, our algorithm leads the ground-breaking $\mathrm{Li}-\mathrm{Li}$ algorithm [11] by a great margin in speed.

First, consider the polynomial system $P(\mathbf{x})=\left(p_{1}(\mathbf{x}), \ldots, p_{n}(\mathbf{x})\right)$ with support

$$
\mathcal{S}_{1}=\cdots=\mathcal{S}_{n}:=\left\{\left(a_{1}, \ldots, a_{n}\right) \mid a_{i} \in\{0,1\}, i=1, \ldots, n\right\}
$$

Obviously, each $\mathcal{S}_{i}$ contains $2^{n}$ points for $i=1, \ldots, n$ and they are all noninterior points. We may regard this system as:

(a) A fully mixed system:

Even though all the supports $\mathcal{S}_{i}, i=1, \ldots, n$, are the same, this special structure will be totally ignored during the mixed volume computation.

(b) A fully unmixed system:

Equality of the supports is fully recognized when the mixed volume is computed.

(c) A semi-mixed system of type $(1, \ldots, 1,\lceil n / 2\rceil)$, where $\lceil\cdot\rceil$ is the standard ceiling function (i.e., $\lceil y\rceil=m$ if $m-1<y \leq m$ ):

Only recognizes the last $\lceil n / 2\rceil$ supports being equal.

The CPU times of our algorithm on each case are shown in Table 2. All the computation was carried out on a $550 \mathrm{MHz}$ Intel Pentium III CPU with $768 \mathrm{Mb}$ of RAM, running Redhat Linux 6.0.

When this system is regarded as a fully mixed system without noticing the equalities of the supports, we list in Table 3 the comparison of the CPU times of our algorithm with those of the $\mathrm{Li}-\mathrm{Li}$ algorithm. As we mentioned before, when applied to fully mixed systems, the major advance in our algorithm compared with the $\mathrm{Li}-\mathrm{Li}$ algorithm is the great amount of cumulative removals of the extraneous constraints, which depends 
heavily in the number of terms in the system. We therefore also list those numbers in the table.

There are other algorithms specially motivated and designed for the computation of mixed volumes of fully unmixed systems, such as

(1) the Dynamic Lifting method given in [17], which has been successfully implemented as a module in PHC [16];

(2) the G-L algorithm given in [6].

While those algorithms may not be effectively applicable to general semi-mixed systems, they are well capable of fully utilizing the characteristic of the equal supports. We list in Table 4 the comparison of the CPU times of our algorithm with those algorithms on this system when it is regarded as a fully unmixed system.

Secondly, we consider the widely considered notoriously difficult benchmark systems, the cyclic- $n$ root problem [5]: $P(\mathbf{x})=\left(p_{1}(\mathbf{x}), \ldots, p_{n}(\mathbf{x})\right)$ with $\mathbf{x}=\left(x_{1}, \ldots, x_{n}\right)$, where

$$
\begin{aligned}
p_{1}(\mathbf{x}) & =x_{1}+x_{2}+\cdots+x_{n-1}+x_{n}, \\
p_{2}(\mathbf{x}) & =x_{1} x_{2}+x_{2} x_{3}+\cdots+x_{n-1} x_{n}+x_{n} x_{1}, \\
p_{3}(\mathbf{x}) & =x_{1} x_{2} x_{3}+x_{2} x_{3} x_{4}+\cdots+x_{n-1} x_{n} x_{1}+x_{n} x_{1} x_{2}, \\
& \vdots \\
p_{n}(\mathbf{x}) & =x_{1} x_{2} \cdots x_{n}-1 .
\end{aligned}
$$

Here,

$$
\begin{aligned}
\mathcal{S}_{1} & =\{(1,0,0,0, \ldots, 0),(0,1,0,0, \ldots, 0), \ldots,(0,0, \ldots, 0,1)\}, \\
\mathcal{S}_{2} & =\{(1,1,0,0, \ldots, 0),(0,1,1,0, \ldots, 0), \ldots,(1,0, \ldots, 0,1)\}, \\
\mathcal{S}_{3} & =\{(1,1,1,0, \ldots, 0), \ldots,(1,1,0, \ldots, 0,1)\}, \\
& \vdots \\
\mathcal{S}_{n} & =\{(1,1, \ldots, 1,1), \ldots,(0,0, \ldots, 0,0)\} .
\end{aligned}
$$

This system is fully mixed, and we list in Table 5 the comparison of our algorithm in CPU time with the $\mathrm{Li}-\mathrm{Li}$ algorithm.

As a reference, we quote the results in [11] which illustrated the superiority of the Li-Li algorithm over the existing codes MVLP [4], [5] and PHC [16] for fully mixed systems in Table 6. As stated in [11], those computations were carried out on a $400 \mathrm{MHz}$ Intel Pentium II CPU with $256 \mathrm{Mb}$ of RAM, running Sun OS 5.6.

In [3], all isolated solutions of the cyclic- $n$ root problems with $n$ up to 12 were numerically located by exploring the special structures of the polynomial systems and the parallel nature of the polyhedral homotopy continuation method.

Finally, we consider the nine-point problem in mechanism design: finding all four-bar linkages whose coupler curve passes through nine prescribed points. The system given in [18] for this problem can be formulated as a fully unmixed system of dimension eight, and its support $\mathcal{S}^{(1)}$ contains 239 points with 150 noninterior points. We list the results involved in Table 7. 
Table 2. Effect of semi-mixed structures.

\begin{tabular}{lrccc}
\hline System & Mixed volume & Fully mixed & $(1, \ldots, 1,\lceil n / 2\rceil)$ & Fully unmixed \\
\hline$n=5$ & 120 & $0.24 \mathrm{~s}$ & $0.09 \mathrm{~s}$ & $0.01 \mathrm{~s}$ \\
$n=6$ & 720 & $3.96 \mathrm{~s}$ & $1.78 \mathrm{~s}$ & $0.12 \mathrm{~s}$ \\
$n=7$ & 5,040 & $1 \mathrm{~m} 16.75 \mathrm{~s}$ & $24.21 \mathrm{~s}$ & $1.30 \mathrm{~s}$ \\
$n=8$ & 40,320 & $30 \mathrm{~m} 13.4 \mathrm{~s}$ & $12 \mathrm{~m} 49.4 \mathrm{~s}$ & $18.3 \mathrm{~s}$ \\
$n=9$ & 362,880 & $12 \mathrm{~h} 10 \mathrm{~m} 3 \mathrm{~s}$ & $3 \mathrm{~h} 59 \mathrm{~m} 49 \mathrm{~s}$ & $4 \mathrm{~m} 45.68 \mathrm{~s}$ \\
\hline
\end{tabular}

Table 3. Effect of the removal of extraneous constraints.

\begin{tabular}{lrrccc}
\hline System & $\begin{array}{r}\text { Number } \\
\text { of terms }\end{array}$ & $\begin{array}{r}\text { Mixed } \\
\text { volume }\end{array}$ & $\begin{array}{c}\text { Current } \\
\text { algorithm }\end{array}$ & $\begin{array}{c}\text { Li-Li } \\
\text { algorithm }\end{array}$ & $\begin{array}{c}\text { Speed-up } \\
\text { Li-Li/Current algorithm }\end{array}$ \\
\hline$n=5$ & 160 & 120 & $0.24 \mathrm{~s}$ & $1.41 \mathrm{~s}$ & 5.88 \\
$n=6$ & 384 & 720 & $3.96 \mathrm{~s}$ & $1 \mathrm{~m} 23.68 \mathrm{~s}$ & 21.13 \\
$n=7$ & 896 & 5,040 & $1 \mathrm{~m} 16.75 \mathrm{~s}$ & $1 \mathrm{~h} 39 \mathrm{~m} 53.95 \mathrm{~s}$ & 78.10 \\
$n=8$ & 2,048 & 40,320 & $30 \mathrm{~m} 13.4 \mathrm{~s}$ & - & - \\
$n=9$ & 4,608 & 362,880 & $12 \mathrm{~h} 10 \mathrm{~m} 3 \mathrm{~s}$ & - & - \\
\hline
\end{tabular}

Table 4. Fully unmixed case.

\begin{tabular}{lccccc}
\hline & \multicolumn{5}{c}{ System } \\
\cline { 2 - 6 } & $n=5$ & $n=6$ & $n=7$ & $n=8$ & $n=9$ \\
\hline Current algorithm & $0.01 \mathrm{~s}$ & $0.12 \mathrm{~s}$ & $1.30 \mathrm{~s}$ & $18.3 \mathrm{~s}$ & $4 \mathrm{~m} 45.68 \mathrm{~s}$ \\
PHC [17] & $0.09 \mathrm{~s}$ & $1.22 \mathrm{~s}$ & $21.38 \mathrm{~s}$ & $7 \mathrm{~m} 34.48 \mathrm{~s}$ & $3 \mathrm{~h} 38 \mathrm{~m} 31 \mathrm{~s}$ \\
$\quad$ Speed-up & & & & & \\
$\quad$ PHC/Current algorithm & 9 & 10.16 & 16.45 & 24.83 & 45.89 \\
$\quad$ G-L Algorithm [6] & $0.005 \mathrm{~s}$ & $0.06 \mathrm{~s}$ & $0.84 \mathrm{~s}$ & $30.79 \mathrm{~s}$ & - \\
$\quad$ Speed-up & & & & & \\
$\quad$ G-L Algorithm/Current algorithm & 0.1 & 0.1 & 0.65 & 1.68 & - \\
\hline
\end{tabular}

Table 5. The cyclic- $n$ problems.

\begin{tabular}{lcrccc}
\hline System & $\begin{array}{c}\text { Number } \\
\text { of terms }\end{array}$ & $\begin{array}{r}\text { Mixed } \\
\text { volume }\end{array}$ & $\begin{array}{c}\text { Current } \\
\text { algorithm }\end{array}$ & $\begin{array}{c}\text { Li-Li } \\
\text { algorithm }\end{array}$ & $\begin{array}{c}\text { Speed-up } \\
\text { Li-Li/Current algorithm }\end{array}$ \\
\hline Cyclic-8 & 58 & 2,560 & $0.44 \mathrm{~s}$ & $0.86 \mathrm{~s}$ & 1.95 \\
Cyclic-9 & 74 & 11,016 & $3.34 \mathrm{~s}$ & $7.86 \mathrm{~s}$ & 2.35 \\
Cyclic-10 & 92 & 35,940 & $27.01 \mathrm{~s}$ & $1 \mathrm{~m} 8 \mathrm{~s}$ & 2.52 \\
Cyclic-11 & 112 & 184,756 & $4 \mathrm{~m} 29 \mathrm{~s}$ & $11 \mathrm{~m} 45 \mathrm{~s}$ & 2.62 \\
Cyclic-12 & 134 & 500,352 & $35 \mathrm{~m} 30 \mathrm{~s}$ & $1 \mathrm{~h} 31 \mathrm{~m} 58 \mathrm{~s}$ & 2.59 \\
Cyclic-13 & 158 & $2,704,156$ & $6 \mathrm{~h} 31 \mathrm{~m} 27.4 \mathrm{~s}$ & $17 \mathrm{~h} 3 \mathrm{~m} 56 \mathrm{~s}$ & 2.62 \\
\hline
\end{tabular}


Table 6. The cyclic- $n$ problems.

\begin{tabular}{lccccc}
\hline System & $\begin{array}{c}\text { Li-Li } \\
\text { algorithm }\end{array}$ & MVLP & $\begin{array}{c}\text { Speed-up } \\
\text { MVLP/Li-Li }\end{array}$ & PHC & $\begin{array}{c}\text { Speed-up } \\
\text { PHC/Li-Li }\end{array}$ \\
\hline Cyclic-8 & $1.23 \mathrm{~s}$ & $35 \mathrm{~s}$ & 28.46 & $49 \mathrm{~s}$ & 39.84 \\
Cyclic-9 & $12.21 \mathrm{~s}$ & $5 \mathrm{~m} 54 \mathrm{~s}$ & 28.99 & $12 \mathrm{~m} 01 \mathrm{~s}$ & 59.05 \\
Cyclic-10 & $1 \mathrm{~m} 36.12 \mathrm{~s}$ & $45 \mathrm{~m} 52 \mathrm{~s}$ & 28.63 & $2 \mathrm{~h} 31 \mathrm{~m} 2 \mathrm{~s}$ & 94.28 \\
Cyclic-11 & $18 \mathrm{~m} 12.74 \mathrm{~s}$ & $7 \mathrm{~h} 24 \mathrm{~m} 40 \mathrm{~s}$ & 24.42 & - & - \\
Cyclic-12 & $2 \mathrm{~h} 14 \mathrm{~m} 25 \mathrm{~s}$ & - & - & - & - \\
Cyclic-13 & $28 \mathrm{~h} 3 \mathrm{~m} 5 \mathrm{~s}$ & - & - & - & - \\
\hline
\end{tabular}

Table 7. The nine-point problem.

\begin{tabular}{|c|c|c|}
\hline $\begin{array}{l}\text { Number of terms } \\
\qquad(150 \times 8)\end{array}$ & $\begin{array}{l}\text { Mixed volume } \\
\quad(79,135)\end{array}$ & CPU time \\
\hline \multicolumn{2}{|c|}{ Current algorithm } & $9.24 \mathrm{~s}$ \\
\hline \multicolumn{2}{|c|}{ G-L algorithm [6] } & $8.34 \mathrm{~s}$ \\
\hline \multicolumn{2}{|c|}{$\begin{array}{l}\text { PHC (dynamic lifting) } \\
\text { Li-Li algorithm }\end{array}$} & $2 \mathrm{~m} 51 \mathrm{~s}$ \\
\hline \multicolumn{2}{|c|}{$\begin{array}{c}\text { (regard the system as fully mixed) } \\
\text { Current algorithm }\end{array}$} & $73 \mathrm{~h} 22 \mathrm{~m} 15 \mathrm{~s}$ \\
\hline \multicolumn{2}{|c|}{ (regard the system as fully mixed) } & $16 \mathrm{~m} 16.06 \mathrm{~s}$ \\
\hline
\end{tabular}

This extreme case strongly illustrates the importance of recognizing the special support structure when the mixed volume is computed. On the other hand, the rather large amount of terms involved in this example also demonstrates the severe effect of the removal of extraneous constraints.

\section{References}

1. D. N. Bernshtein (1975), The number of roots of a system of equations, Functional Anal. Appl., 9(3), 183-185. Translated from Funktsional. Anal. i Prilozhen., 9(3), 1-4.

2. M. J. Best and K. Ritter (1985), Linear Programming: Active Set Analysis and Computer Programs, Prentice-Hall, Englewood Cliffs, NJ.

3. Y. Dai, S. Kim, and M. Kojima, Computing all nonsingular solutions of cyclic- $n$ polynomial using polyhedral homotopy continuation methods, Technical Report B-373, available at http://www.is.titech. ac.jp/ kojima/sdp.html.

4. I. Z. Emiris and J. F. Canny (1993), A practical method for the sparse resultant, Proceedings of the 1993 International Symposium on Symbolic Computation, ACM, New York, pp. 183-192.

5. I. Z. Emiris and J. F. Canny (1995), Efficient incremental algorithms for the sparse resultant and the mixed volume, J. Symbolic Comput., 20, 117-149.

6. T. Gao and T. Y. Li (2000), Mixed volume computation via linear programming, Taiwan J. Math., 4, 599-619.

7. B. Huber and B. Sturmfels (1995), A polyhedral method for solving sparse polynomial systems, Math. Сотр., 64, 1541-1555.

8. B. Huber and B. Sturmfels (1997), Bernshtein's theorem in affine apace, Discrete Comput. Geom., 17(2), $137-141$ 
9. T. Y. Li (1997), Numerical solution of multivariate polynomial systems by homotopy continuation methods, ACTA Numer., 399-436.

10. T. Y. Li (1999), Solving polynomial systems by polyhedral homotopies, Taiwan J. Math., 3, 251-279.

11. T. Y. Li and X. Li (2001), Finding mixed cells in the mixed volume computation, Found. Comput. Math., 1, 161-181.

12. T. Y. Li and X. Wang (1997), The BKK root count in $\mathbb{C}^{n}$, Math. Comp, 65(216), 1477-1484.

13. J. M. Rojas (1994), A convex geometric approach to counting the roots of a polynomial system, Theoret. Comput. Sci., 133, 105-140.

14. J. M. Rojas and X. Wang (1996), Counting affine roots of polynomial systems via pointed Newton polytopes, J. Complexity, 12, 116-133.

15. A. Takeda, M. Kojima, and K. Fujisawa, Enumeration of all solutions of a combinatorial linear inequality system arising from the polyhedral homotopy continuation method, Preprint.

16. J. Verschelde (1999), Algorithm 795: PHCPACK: a general-purpose solver for polynomial systems by homotopy continuation, ACM Trans. Math. Software, 25, 251-276.

17. J. Verschelde, K. Gatermann, and R. Cools (1996), Mixed-volume computation by dynamic lifting applied to polynomial system solving, Discrete Comput. Geom., 16(1), 69-112.

18. C. W. Wampler, A. P. Morgan, and A. J. Sommese (1992), Complete solution of the nine-point path synthesis problem for four-bar linkages, ASME J. Mech. Design, 114, 153-159.

Received September 5, 2001, and in revised form March 1, 2002. Online publication November 14, 2002. 